\title{
Prior context influences motor brain areas in an auditory oddball task and prefrontal cortex multitasking modelling
}

Carlos A. Mugruza-Vassallo ${ }^{1 *}$ (DD, Douglas D. Potter ${ }^{2}$, Stamatina Tsiora ${ }^{3}$, Jennifer A. Macfarlane ${ }^{4}$ and Adele Maxwell ${ }^{2}$

\begin{abstract}
In this study, the relationship of orienting of attention, motor control and the Stimulus- (SDN) and Goal-Driven Networks (GDN) was explored through an innovative method for $\mathrm{fMRl}$ analysis considering all voxels in four experimental conditions: standard target (Goal; $\mathrm{G}$ ), novel (N), neutral (Z) and noisy target (NG). First, average reaction times (RTs) for each condition were calculated. In the second-level analysis, 'distracted' participants, as indicated by slower RTs, evoked brain activations and differences in both hemispheres' neural networks for selective attention, while the participants, as a whole, demonstrated mainly left cortical and subcortical activations. A context analysis was run in the behaviourally distracted participant group contrasting the trials immediately prior to the $G$ trials, namely one of the Z, N or NG conditions, i.e. Z.G, N.G, NG.G. Results showed different prefrontal activations dependent on prior context in the auditory modality, recruiting between 1 to 10 prefrontal areas. The higher the motor response and influence of the previous novel stimulus, the more prefrontal areas were engaged, which extends the findings of hierarchical studies of prefrontal control of attention and better explains how auditory processing interferes with movement. Also, the current study addressed how subcortical loops and models of previous motor response affected the signal processing of the novel stimulus, when this was presented laterally or simultaneously with the target. This multitasking model could enhance our understanding on how an auditory stimulus is affecting motor responses in a way that is self-induced, by taking into account prior context, as demonstrated in the standard condition and as supported by Pulvinar activations complementing visual findings. Moreover, current $\mathrm{BCl}$ works address some multimodal stimulusdriven systems.
\end{abstract}

Keywords: Attention, Cognitive modelling, Electroencephalography (EEG), Event-related potential (ERP), Executive function, Cue-target onset asynchrony (CTOA), Functional magnetic resonance imaging (fMRI), Motor networks, Multitask applications, Prefrontal cortex (PFC), Orienting of attention, Running average reaction times

\section{Introduction}

Recent works in stimulus-driven neural networks and learning systems are awakening the interest in multimodal attention systems, such as works in Brain

\footnotetext{
*Correspondence: cmugruza@yahoo.com

${ }^{1}$ Grupo de Investigación de Computación Y Neurociencia Cognitiva, Facultad de Ingeniería Y Gestión, Universidad Nacional Tecnológica de Lima Sur - UNTELS, Lima, Perú

Full list of author information is available at the end of the article
}

Computer Interface $(\mathrm{BCI})$ systems in both visual and auditory modalities $[1,2]$, also considering tasks with multiple conditions [3]. In the present work, the interaction of the auditory and motor systems is studied and modelled using an odd/even auditory number decision task, whilst performing simultaneous scalp electroencephalography (EEG) and functional magnetic resonance imaging (fMRI) recordings. The effects of prior context on attention have traditionally been studied with fMRI in visual tasks. Koechlin and colleagues
Springer Open

(c) The Author(s) 2021. This article is licensed under a Creative Commons Attribution 4.0 International License, which permits use, sharing adaptation, distribution and reproduction in any medium or format, as long as you give appropriate credit to the original author(s) and the source, provide a link to the Creative Commons licence, and indicate if changes were made. The images or other third party material in this article are included in the article's Creative Commons licence, unless indicated otherwise in a credit line to the material. If material is not included in the article's Creative Commons licence and your intended use is not permitted by statutory regulation or exceeds the permitted use, you will need to obtain permission directly from the copyright holder. To view a copy of this licence, visit http://creativeco mmons.org/licenses/by/4.0/. 
$[4,5]$ used an experimental task in which participants were asked to discriminate coloured shapes or letters, or ignore a non-goal stimulus, on the basis of an instruction cue that initiated each block. Based on their findings, they suggested that the lateral frontal lobes contribute to a cascade of control processes mediating sensory, contextual, and episodic control, implemented in premotor, caudal and rostral lateral prefrontal cortical regions, respectively [4]. Therefore, pending behavioural responses are maintained and managed by prefrontal areas, and the activation of frontal areas can be affected by multitasking. On the other hand, it has been previously shown [3] that in an auditory oddball task with 4 conditions where participants had to maintain a number parity decision goal while ignoring novel distractors, stimulus properties (here features) and previous context were covariates that helped to understand and explain attention switching. Furthermore, it was shown that these findings were not related to the time intervals between stimuli, as measured by the P300. Their study pointed to single-trial event-related potential (ERP) dependence on prior signals; the longer the signals in time, the fewer effects mismatch negativity $(\mathrm{MMN})$ and the stimulus features explained about the variance of the P300 amplitude. Moreover, a similar study that used linear filtering suggested a use for a schizophrenia therapy study of attention and executive functions [6]. The addition of fMRI measures in this type of research would be beneficial in better understanding how prior context influences behavioural response in the auditory modality.

Current theories of attention assume the involvement of a distributed control network of areas in stimulusdriven selection of the behaviourally relevant information [7]. Furthermore, these control networks share common areas and interact with the goal-driven network (GDN) (see review of the fronto-parietal visual attention network using single-cell recordings in monkeys and fMRI in humans by Kastner and Ungerleider [8]). Moreover, the actor critic architecture for learning and execution proposed by Savalia and colleagues [9] where time and hierarchical management of sequence induce different work at loop on basal ganglia-frontal cortex and hippocampus-frontal cortex. While other studies were seeking how motor responses were done differently for every participant in a decision-making task [10] and how the reference frame is important for decisions in hand choice [11]. Even more, on goal-driven tasks behavioural motor answers that used greater auditory processing suppress responses in the auditory cortex [12] and recent report has shown motor-dependent changes in auditory cortical dynamics were driven by a subset of neurons in the secondary motor cortex that innervate the auditory cortex and are active during movement [13]. These studies have led to the uncertainty of how the auditory stimulus is affecting motor responses in a kind of self-induced motor signal [14]. The present work has looked at motor responses and their relation with prefrontal areas.

Wagner and colleagues used a word goal decision task to find how some tasks are recognized or not in the human brain. The goal was a semantic signal (abstract or concrete) and a non-semantic signal (upper or lower-case letter). Results pointed to the lateralization response for the left prefrontal cortex, left fusiform gyrus and temporal cortices [9]. However, this study focused on explaining how lateralization activations may be changing in time for each stimulus type.

Few studies have explored the generators of auditory novelty using EEG and fMRI measures. Opitz and colleagues [15] used a block design in an auditory oddball task, where the goal standard stimulus was a tone of $600 \mathrm{~Hz}(83.4 \%)$, the non-goal deviant stimulus was a tone of $1000 \mathrm{~Hz}(8.3 \%)$ and the non-goal novel stimulus was an environmental sound. They found that novel sounds activated the superior parietal cortex and those subjects showing strong N4 deflections showed an additional right prefrontal cortex (rPFC) activation [15]. Bearing in mind the distributed areas for attention [7], Strobel and colleagues aimed to improve Opitz and colleagues [15] study using simultaneous EEG/fMRI recordings with an event-related design in an auditory oddball task. They used tones of 350 and $650 \mathrm{~Hz}$ and environmental sounds where participants were required to silently count standard tones as targets in $50 \%$ of the cases and novel sounds as targets in the other $50 \%$. They found that the bilateral superior temporal and right inferior frontal areas showed strongest activation with novel sounds [16].

Kiehl and colleagues used fMRI to study the brain areas activated in an auditory oddball task seeking to answer whether gender influences the magnitude or distribution of brain activity associated with the P3a and P3b responses. They implemented a task in which the standard tone stimulus had a probability of 0.8 , the target tone stimulus had a probability of 0.1 and the novel stimuli had a probability of 0.1 with an InterTrial Interval (ITI) of $2000 \mathrm{~ms}$. They examined hemodynamic fMRI responses of target detection and novel stimulus processing in five groups of 20 subjects. They did not find evidence of a gender effect, but this study is relevant to the present research because it was an oddball task, and the ITI was similar. We used a single sound per trial and gender was imbalanced. They found around 28 brain areas for the target over the standard stimulus (the superior parts of the left PreCentral Gyrus, left middle and Inferior Frontal Gyrus, and 
brainstem), 20 brain areas for the novel over the nongoal standard tone stimulus (bilateral Amygdala, Anterior and Posterior Cingulate, bilateral inferior parietal lobe, and brainstem), 29 brain areas for the target over the novel stimulus (bilateral middle Frontal Gyrus, right Inferior Frontal Gyrus, left PreCentral and postcentral Gyrus, and right Cerebellum) and 29 brain areas for the target over the novel stimulus (bilateral middle Frontal Gyrus, bilateral middle Temporal Gyrus, and right precentral Gyrus and additional regions in left middle frontal Gyrus, right middle temporal Gyrus, and left angular Gyrus and Precuneus) [17]. Therefore, in terms of comparison we should expect to find several areas activated for the four switching conditions.

Nowadays, mixed modalities are tough to cognitive robotics, many works employ image recognition in combination to motor answers due to its many potential applications. For example Zeng and colleagues, employ several paths (Somatosensory Input $\rightarrow$ Thalamus $\rightarrow$ Primary somatosensory cortex $\rightarrow$ SMG), the visual ventral stream (Visual Input $\rightarrow$ Thalamus $\rightarrow$ Primary visual cortex $\rightarrow$ EBA/OFA $\rightarrow$ FBA/ FFA $\rightarrow$ ITG $\rightarrow$ SMG), and the visual dorsal stream [Visual Input $\rightarrow$ Thalamus $\rightarrow$ Primary visual cortex $\rightarrow$ (MT/ V5, EBA/OFA, FBA/FFA) $\rightarrow$ STS $\rightarrow$ SMG [18] without employing auditory components for cognitive tasks.

On the one hand, some multisensorial modalities, such as visuo-haptic object recognition are now as multimodal interactions take place between the two sensory modalities [12]. Evolutionary multitasking was recently developed through algorithms seeking brain function. This multi-X evolutionary computation is based on multiobjective optimization problems (MOPs) employing frequency or objective functions ( $f$ ) for vectors of decision variables $(y)$ in the search space $(Y)$ [19] following (1):

$$
\operatorname{maximize}(y \in Y) f(y)=\left|f_{1}(y) ; f_{2}(y) ; \ldots ; f_{K}(y)\right| .
$$

Then, for $K$ different tasks (T1, T2,... TK) the MOP in terms of the populations, multitask would follow (2), having $\Sigma w j k=1, \forall k$; and $w j k \geq 0 ; \forall j ; k$

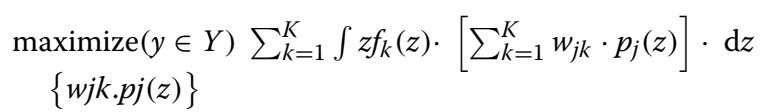

The aims of the present analysis and modelling on the present work are to determine if the simultaneous EEG and fMRI recordings can provide insights into (a) the effect of prior stimulus contexts across participants; (b) the sources of the generators of the positive deflections in the ERP waveforms, including the smaller right lateralized positive deflection observed to novel sounds; (c) the modelling of multimodal stimulus-driven network for practical use.

Based on the findings of the literature summarized above and the results of four task switching [3], the following hypotheses were drawn for an experiment to develop a better approach for modelling:

H1: The participants must orient their attention in response to novel distractors and this should be associated with bilateral activations of the goal-driven system. This would confirm the sensitivity of the task in the framework of the distributed control of attention proposed by Corbetta and colleagues [ 7,20$]$.

$\mathrm{H} 2$ : Bearing in mind the contextual effect of the immediately previous trial, in a task with several conditions $[4$, 5] several significant different brain areas should appear in different fMRI contrasts. Therefore, based on Koechlin's findings and results in the experiment with 4 conditions [3], the Goal-driven experiment should produce significant modulations of activations in memory areas as a result of modulation by different areas of the prefrontal cortex, dependent of the level of contextually based executive controls outlined by Koechlin et al. [4, 5]. The differing contextual conditions associated with the different experimental conditions are expected to activate different prefrontal areas for Novel followed by the Goal (N.G), simultaneous Novel and Goal followed by the Goal (NG.G) and Zero followed by the Goal (Z.G), i.e. different prefrontal activations should be found in Z.G vs. G.G, N.G vs. G.G, NG.G vs. Z.G, NG.G vs. G.G, and N.G vs. Z.G contrasts.

H3: Auditory modelling may be better defined over motor control through modelling at multitask cognitive computation.

\section{Methods}

\subsection{Participants}

Twelve adults participated in the present study (mean age: $30.75 \pm 8.8$ years; range $18-48$ years). All subjects self-reported normal hearing and no history of known neurological illness. The study was approved by the University of Dundee Institutional Review Board and NHS Tayside and was performed in accordance with the ethical standards for radiology intervention by NHS Tayside. All participants gave informed written consent before participating in the study. One healthy participant was excluded because the structural MRI was lost, leaving 11 healthy (10 right-handed) subjects.

\subsection{Experimental design}

Subjects were asked to perform an odd/even auditory number decision task during simultaneous scalp EEG and fMRI recordings. The paradigm was composed of 400 trials, with trials chosen pseudo-randomly from one of four 
Table 1 Stimuli combinations for the simultaneous EEG/fMRI experiment

\begin{tabular}{lclll}
\hline Stimuli name & Number of presentations & Code processed & & Stimuli \\
\cline { 4 - 5 } & & & S2 & Type \\
\hline Standard goal stimuli & & & Number & $300 \mathrm{~ms}$ \\
Non-goal stimuli & 250 & $\mathrm{Z}$ & Zero & $200 \mathrm{~ms}$ \\
Simultaneous novel and goal & 50 & $\mathrm{NG}$ & Number + Novel & $300 \mathrm{~ms}$ \\
Novel stimuli & 50 & $\mathrm{~N}$ & Novel & $55,135,200 \mathrm{~ms}$
\end{tabular}

SOA stimulus-onset asynchrony

different conditions. Each trial consisted of a sound stimulus. The parameters of the stimuli are given in Table 1 . Participants were asked to respond by pressing a button as quickly as possible without sacrificing accuracy. Participants used the index and middle fingers of their right hand. The Inter-Trial Interval (ITI) was between 1900 and $2100 \mathrm{~ms}$. The task was presented in one single block (400 trials) with each of the four conditions presented in random order. Stimulus sequence was the same across all participants.

\subsection{Stimuli}

Stimuli were sounds presented using Nordic Neurolab Electrostatic Headphones at $80 \mathrm{~dB}$ sound pressure level. Sound files were stereo with 16-bit resolution and $22,050 \mathrm{~Hz}$ sampling rate.

In the standard goal stimulus condition (G), the stimulus (S2) was a number of 300 ms duration. In the nongoal stimulus condition (Z), S2 was the number zero of 300 ms duration. In the novel only condition (N), S2 was a novel sound of 55, 135 or 200 ms duration. Finally, in the simultaneous novel and goal condition (NG), S2 was a number of $300 \mathrm{~ms}$ duration simultaneously presented with a lateralized novel sound of $100 \mathrm{~ms}$ duration.

\subsection{EEG recording}

EEG data were recorded continuously using a 64-channel EEG acquisition system designed especially for the MR environment (Vision Recorder, Brain Product, Inc., Munich, Germany). The electrode placement followed the extended international $10-20$ system, using $\mathrm{FCz}$ as a reference electrode. Amplified signals were digitized at $5000 \mathrm{~Hz}$ with a 16-bit resolution. All electrode impedances were $<20 \mathrm{k} \Omega$. Data were band-pass filtered between $0.016-250 \mathrm{~Hz}$ during data acquisition. Trials with excessive peak-to-peak deflections, amplifier clipping or excessive high-frequency (EMG) activity were excluded before analysis. This data has provided P300 results across averaging participants, but noise data was not able to combine results with fMRI acquisition.

\section{5 $\mathrm{fMRI}$ acquisition and analysis}

Whole-brain images (30 slices; $2.6 \mathrm{~mm}$ thick, $0.4 \mathrm{~mm}$ gap, $64 \times 64$ pixels in-plane resolution, overall resolution $3.75 \times 3.75 \times 5 \mathrm{~mm}$ ) were collected on a $3-\mathrm{T}$ Trio Siemens scanner using an echo-planar imaging sequence. Scans were acquired with a repetition time of $2.5 \mathrm{~s}$ and echo time of $30 \mathrm{~ms}$. Additionally, a T1-weighted structural scan was acquired for each subject (1 mm isotropic resolution). SPM8 was used for both pre-processing and statistical analysis [21]. Images were spatially realigned to reduce movement artefacts. Mean image and structural data were used for co-registration, and co-registration results were then used to produce normalized images. Images were spatially normalized to the MNI template and spatially smoothed using a Gaussian kernel of $8 \mathrm{~mm}$ full-width at half height. The BOLD signal was then high-pass filtered with a cut-off of $256 \mathrm{~s}$.

A subset of different possible regressors was used: (1) from initial conditions; (2) extended contextual conditions (see Fig. 1). To explore the main effects of conditions and contextual analysis in the whole group, we adopted a voxel-wise type I error threshold of $\alpha=0.03$ and used the cluster extent method to correct for multiple comparisons [22]. Areas exceeding a corrected cluster-wise type I error threshold of $\alpha=0.006(k>1055$ voxels, equivalent in spatial extent to 15 original nonresampled voxels) were selected for further analysis to determine the directionality of category-specific main effects and to test for interactions. Given that the cluster extent method is not as stringent as false discovery rate (FDR) or family wise error (FWE), we have chosen $\alpha=0.03$. With these 1055 voxels, the second-level random effects analyses were conducted. FDR script (https://warwick.ac.uk/fac/sci/statistics/staff/acade mic-research/nichols/software/fdr/fdrm) was conducted on SPM, employing Nichols later Matlab script [23]. These analyses were achieved by entering the six covariate images of interest into one-group t-test. Due to the small number of participants for orienting $(n=6)$ and non-orienting $(n=5)$, only statistical analysis 


\section{Prior context influence motor brain areas activated in an auditory orienting task and use for multitasking modelling}

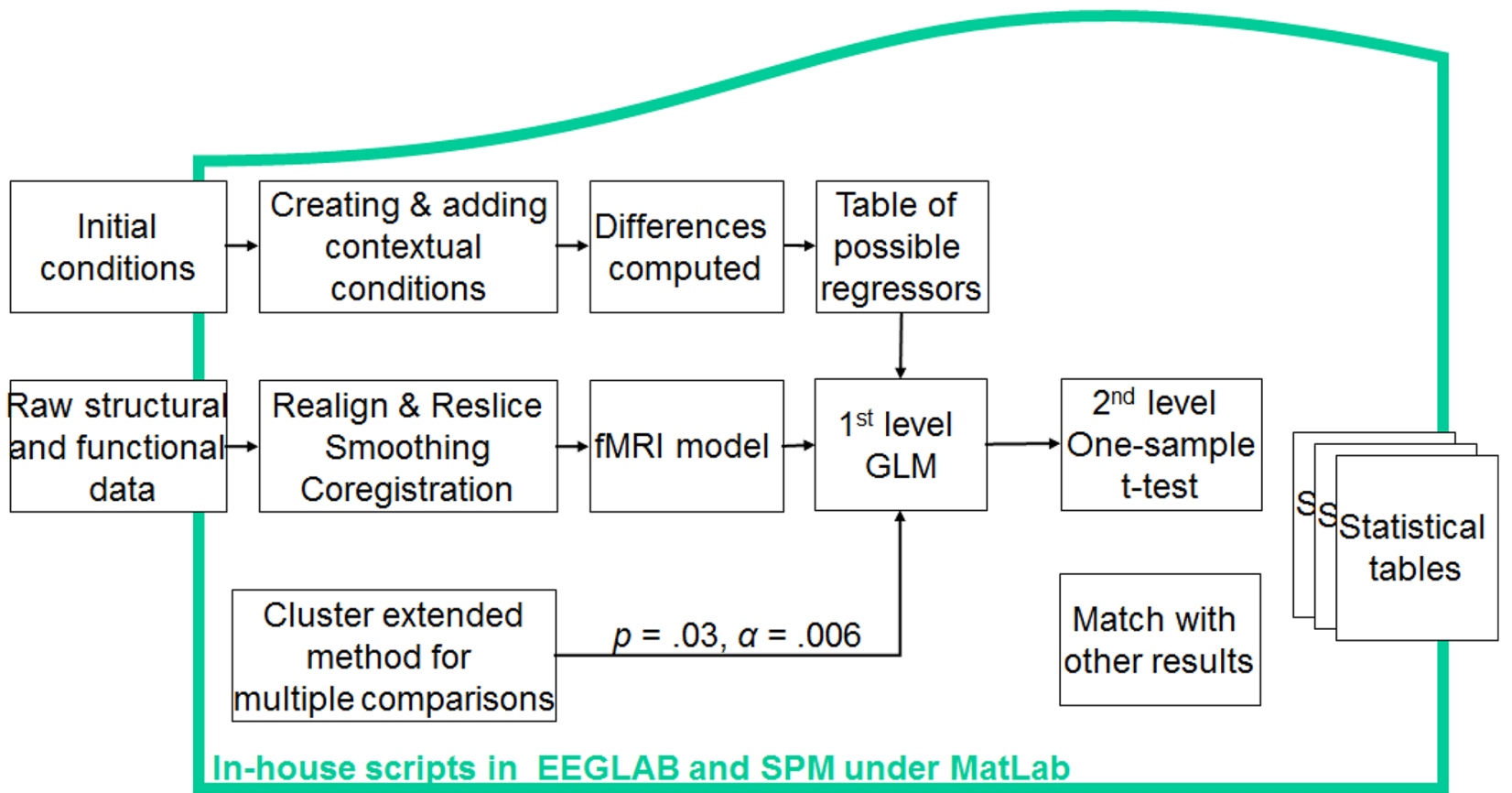

Fig. 1 Preprocessing and analysis diagram used for the auditory oddball task in the simultaneous EEG and fMRI recording

within 'distracted' participants $(n=6)$ and the whole $(n=11)$ groups was carried out.

\subsection{Synthesis for auditory and motor modelling (conclusions)}

Interpretation of results would allow to model the function of auditory and motor function in an auditory oddball task. Therefore, results would allow having a better grasp of motor and auditory interaction on Goal-Driven tasks.

\section{Results}

\subsection{Behavioural results}

Both accuracy and mean response latencies were examined in the critical trials common to our two goal stimulus conditions, Goal (G) and the simultaneous Novel and Goal (NG). Overall, participants performed well (94\% accuracy of goal trials). The proportion of correct responses was analysed using a 2-way ANOVA. The main effect of condition was not significant across subjects $(F(1,11)=0.43, p=0.5136)$.
A time series analysis using a running average of reaction times was conducted in each participant to explore the basis of these non-significant results and the small effect size $(<0.01)$. Running average reaction times in the 12 control participants for conditions G (coloured in black) and NG (coloured in gray) are illustrated in Fig. 2.

Solid lines in the upper plots are the means for every condition (black for standard Goal stimuli, gray for the simultaneous Novel and Goal). In the bottom plots the difference of the RTs between the $\mathrm{G}$ condition minus the NG condition are shown. There the average and standard deviation calculation of reaction times was run, taking as the centre, the central trial plus and minus 75 trials (condition G) or 15 trials (condition NG) across the whole of the possible accurately answered trials (this explains why the measure does not start from 0 and finish at 400) rendering 151 trials (condition G) and 31 trials (condition NG). This is called running average of Reaction time or running average RT.

Novel distractors slowed RTs in 6 participants $(7,8$, $10,14,15$ and 16), speeded up reaction times in 4 participants $(4,5,9$ and 12) while 2 participants (6 and 11) showed no differences. In Fig. 2 the running average RTs 


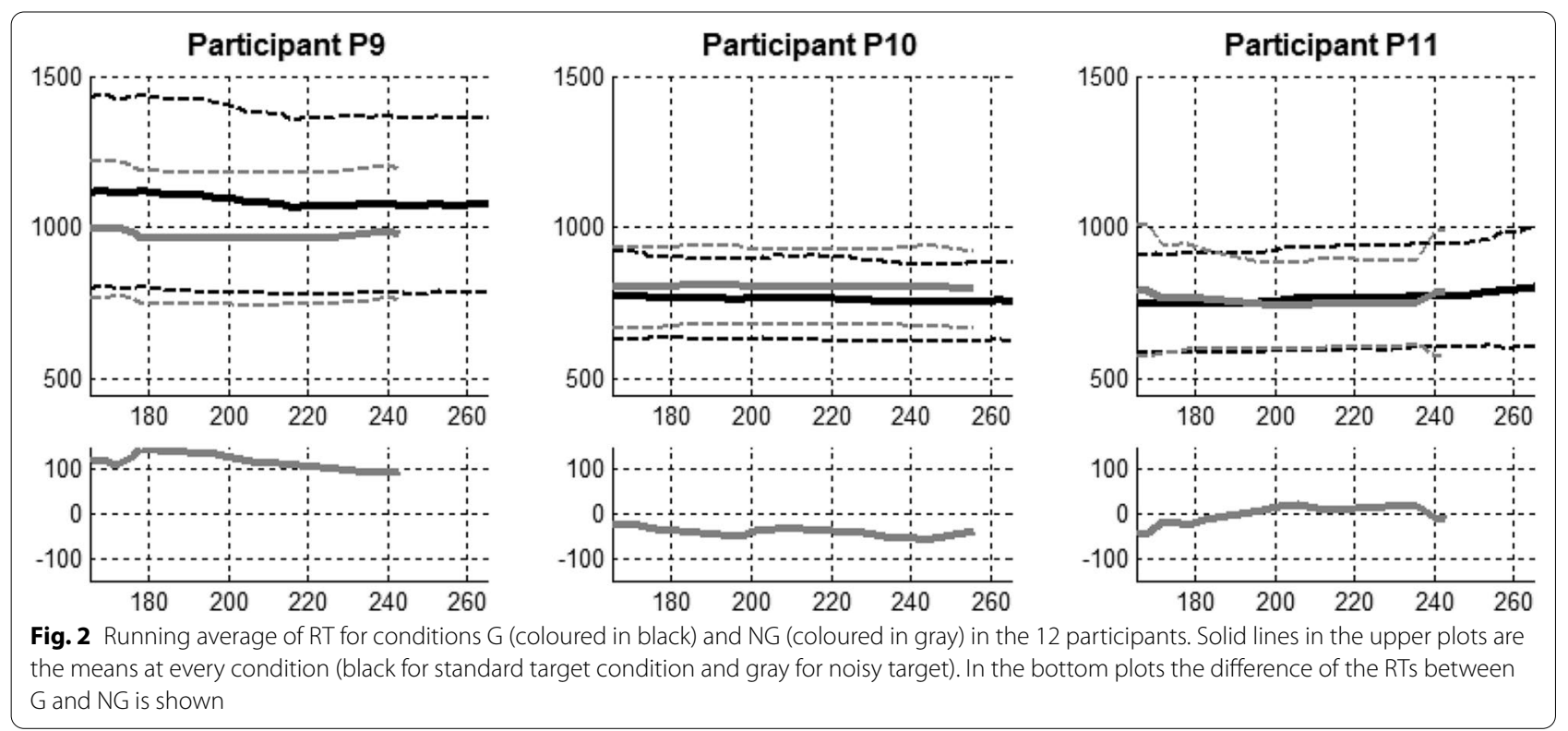

for the G and NG conditions are illustrated along with the average difference between the two conditions.

Overall, the lack of significant differences in RT in the two-way ANOVA may be explained by the individual differences in pattern of the running average reaction times in the different conditions. Some individuals clearly show distraction effects while others do not.

\section{2 fMRI results based on the immediately preceding context analysis included in the analysis for 'distracted' participants}

Continuing with the focus of the condition of the trial immediately prior to the current trial as suggested in controls and schizophrenic participants [3], the classical fMRI analysis was extended. The contextual cases tested in this fMRI analysis were: Z.G vs. G.G, N.G vs. G.G, NG.G vs. Z.G, NG.G vs. G.G, and N.G vs. Z.G.

Common different brain area activations are in the Left Parietal Precuneus, the Right Sub lobar Insula and in the Right Temporal Lobe in the Superior Temporal Gyrus ( $\mathrm{R}$ STG). In the last case, L STG has different brain activation except for the N.G vs. Z.G contrast (this is discussed in Sect. 4.2).

Table 2 lists the differences observed in the contrast between Z.G and G.G. Both hemispheres in frontal, temporal, parietal, occipital and limbic brain areas showed differences strongly biased to the Z.G contextual condition. According to the results, there are no brain areas with the same BA in the positive and negative contrasts, and only the Left Medial Frontal Gyrus with different Brodmann Areas (BA), BA 6 biased to Z.G and the BA 9 biased to the G.G condition. The left and right frontal areas in Inferior and Middle Frontal Gyrus (IFG and MFG) are positive activated. Also, positive differences were found for R MFG, R IFG, and R IPL, and L IPs and R IPs (Fig. 3).

Table 3 lists the differences observed in the contrast of sequences N.G and G.G. Both hemispheres in frontal, temporal, parietal and right limbic brain areas showed differences strongly biased to the N.G contextual condition. According to the results, there are no common areas for positive and negative contrast. There are strong frontal differences in R Precentral Gyrus and the R IFG and in 5 other frontal areas. Results showed that the greatest differences measured occurred towards the most frontal area of the brain, with the greatest frontal differences measuring up to $37 \mathrm{~mm}$ in the left MFG and up to $28 \mathrm{~mm}$ in the right MFG, which means that frontal activation is larger in the left hemisphere when the Novel is presented immediately before the present Goal stimulus. This left lateralization response is consistent with the present Goal stimulus. Figure 4 shows this contrast.

Moreover, the PreCentral Gyrus is activated differently between this N.G and G.G contrast, with a clearly right lateralized bias. Bearing in mind that this area was not found in the results for the N and G contrast, thus the Novel before a Goal makes more contribution to different motor area activations. Therefore, this result suggests that attention to the task by the participants produces different motor control in N vs. G contrast and in N.G and G.G contrast. This is addressed in the discussion. Overall these differences in the Prefrontal Cortex by the trial before the $\mathrm{G}$ condition in analysis support hypothesis $\mathrm{H} 2$. 


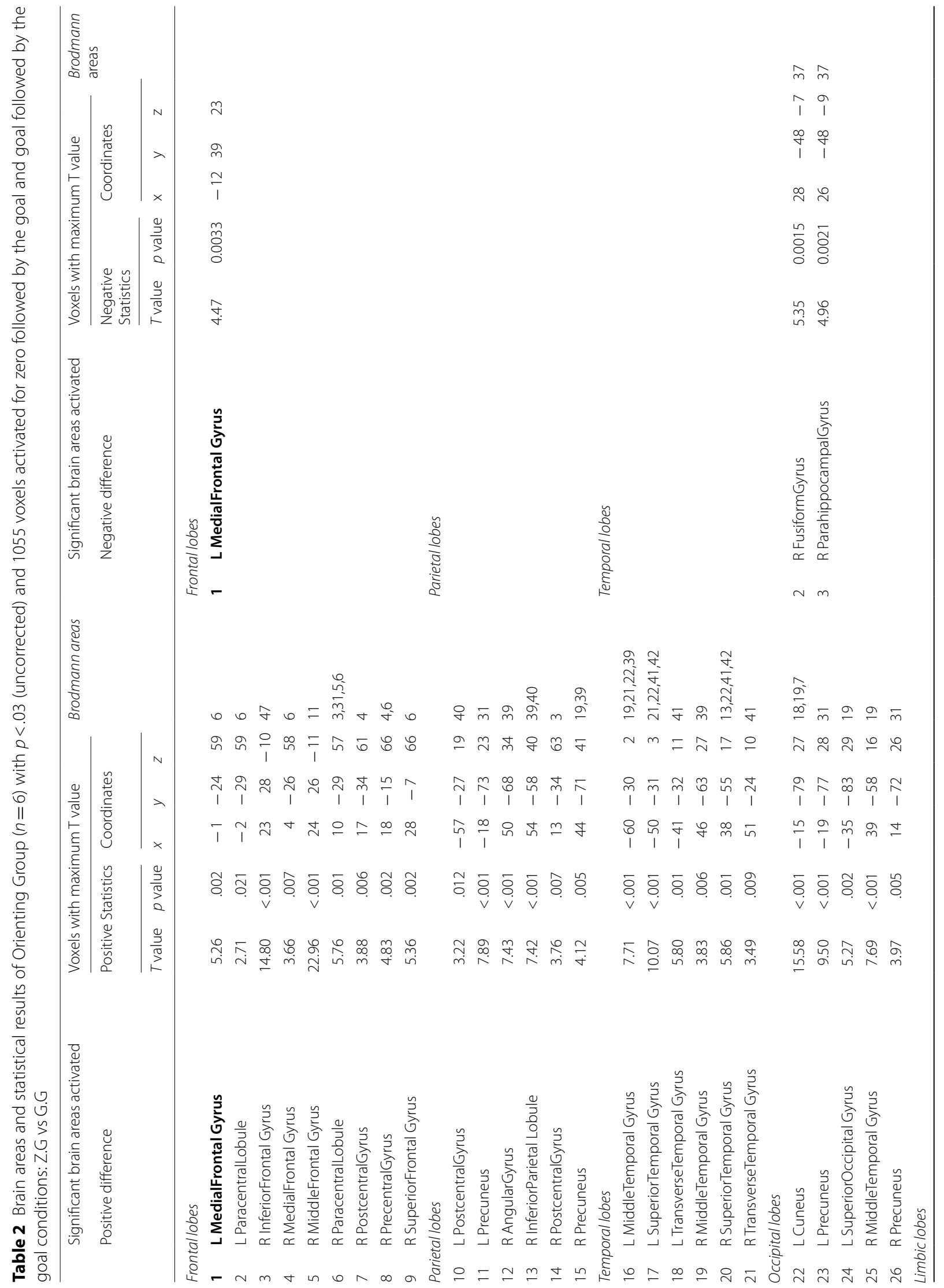




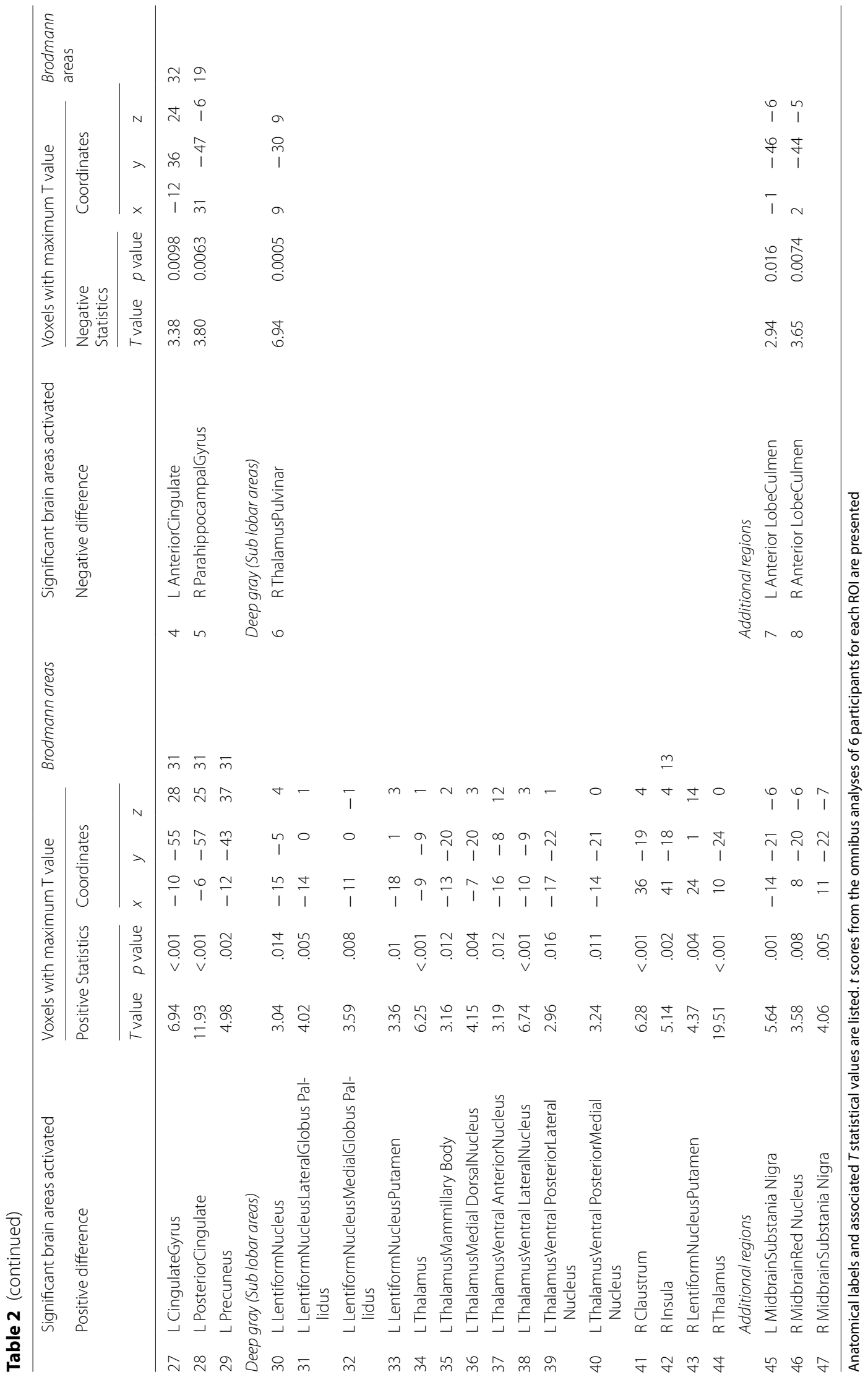


Brain regions for the contrast between sequences N.G and $\mathrm{G} . \mathrm{G}$ as conditions.
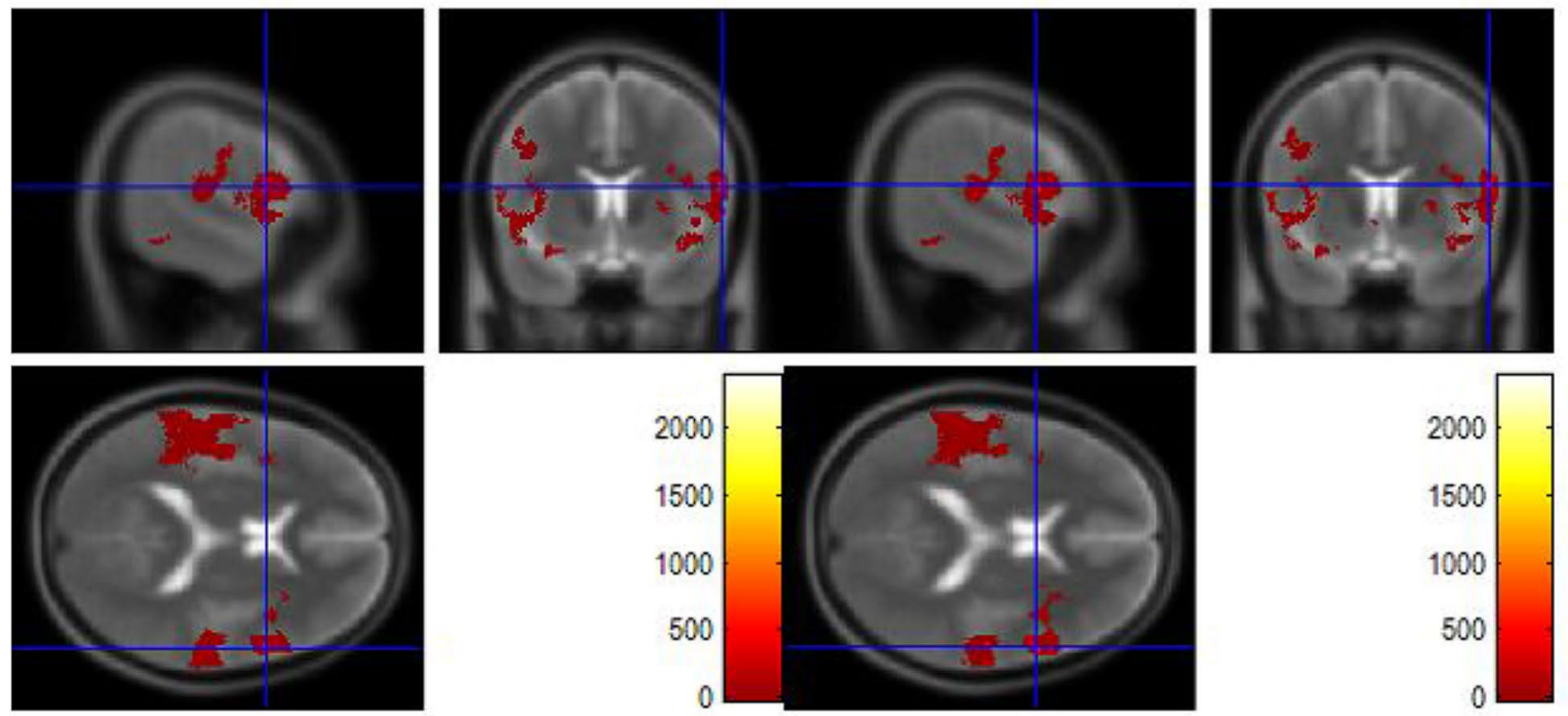

Strongest contrast in the Right Precentral Gyrus [58 8 12]

Strong contrast in the Right Inferior Frontral Gyrus [58 7 16]

Brain regions for the contrast between sequences N.G and NG.G as conditions.
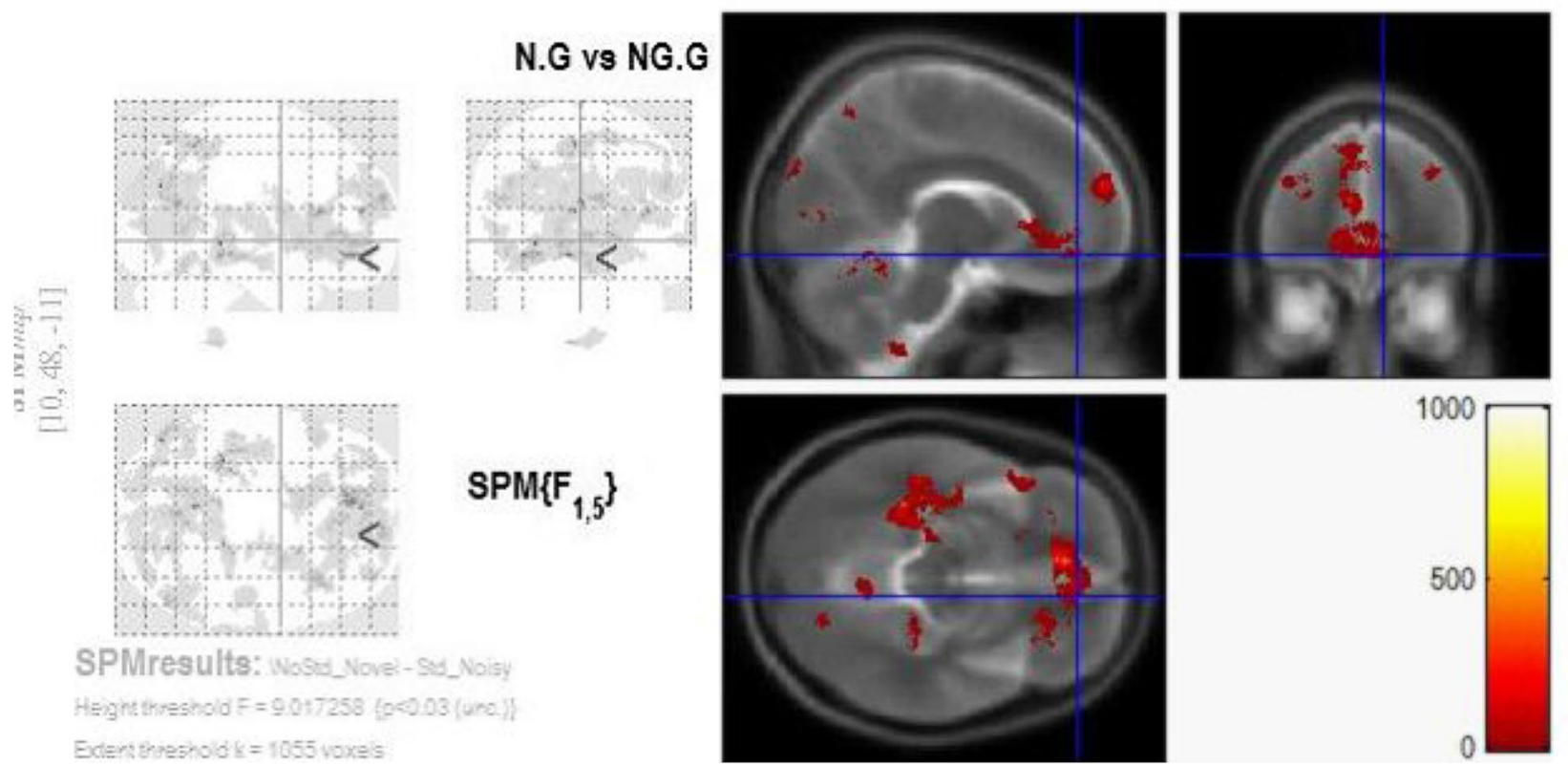

Fig. 3 Cross-sectional images with the blue cross bars point to the maximum F value in brain regions for the contrast between sequences N.G and G.G as conditions on the top and sequences N.G and NG.G as conditions on the bottom

Table 4 lists the observed differences for the contrast of sequences N.G and NG.G, showing frontal differences in 10 regions. Both hemispheres in frontal, temporal, parietal and limbic brain areas showed differences strongly biased to the N.G contextual condition. According to the results, the Right IFG with BA 13, Right 
Table 3 Brain areas and statistical results of Orienting Group $(n=6)$ with $p<.03$ and 1055 voxels activated for novel and goal stimulus vs goal and goal conditions: N.GvsG.G

\begin{tabular}{|c|c|c|c|c|c|c|c|}
\hline & \multirow{3}{*}{$\begin{array}{l}\text { Significant brain areas activated } \\
\text { Positive difference }\end{array}$} & \multicolumn{5}{|c|}{ Voxels with maximum Z score } & \multirow[t]{3}{*}{ Brodmann areas } \\
\hline & & \multicolumn{2}{|l|}{ Statistics } & \multicolumn{3}{|c|}{ Coordinates } & \\
\hline & & Tvalue & $p$ value & $x$ & $y$ & $z$ & \\
\hline \multicolumn{8}{|c|}{ Frontal lobes } \\
\hline 1 & L InferiorFrontal Gyrus & 14.38 & $<.001$ & -42 & 13 & -7 & 47 \\
\hline 2 & L MiddleFrontal Gyrus & 29.22 & $<.001$ & -24 & 37 & -8 & $11,47,6$ \\
\hline 3 & L PrecentralGyrus & 14.09 & $<.001$ & -39 & -18 & 41 & 4 \\
\hline 4 & L SubcallosalGyrus & 4.95 & .002 & -19 & 15 & -11 & 47 \\
\hline 5 & R CingulateGyrus & 6.62 & $<.001$ & 15 & 14 & 37 & 32 \\
\hline 6 & R InferiorFrontal Gyrus & 37.42 & $<.001$ & 57 & 8 & 14 & $44,47,6,9$ \\
\hline 7 & R MedialFrontal Gyrus & 3.26 & .011 & 10 & 28 & 30 & 6,9 \\
\hline 8 & R MiddleFrontal Gyrus & 16.43 & $<.001$ & 53 & 6 & 34 & $6,8,9$ \\
\hline 9 & R PrecentralGyrus & 48.74 & $<.001$ & 58 & 8 & 12 & $4,44,6$ \\
\hline \multicolumn{8}{|c|}{ Parietal lobes } \\
\hline 10 & L InferiorParietal Lobule & 21.01 & $<.001$ & -64 & -24 & 28 & 40 \\
\hline 11 & L PostcentralGyrus & 35.14 & $<.001$ & -61 & -21 & 28 & 2 \\
\hline 12 & L Precuneus & 19.25 & $<.001$ & -13 & -56 & 51 & 7 \\
\hline 13 & R InferiorParietal Lobule & 9.72 & $<.001$ & 66 & -34 & 30 & 40 \\
\hline 14 & R PostcentralGyrus & 5.15 & .002 & 57 & -22 & 43 & 2,3 \\
\hline 15 & R Precuneus & 15.35 & $<.001$ & 18 & -58 & 55 & 7 \\
\hline \multicolumn{8}{|c|}{ Temporal lobes } \\
\hline 16 & L FusiformGyrus & 18.68 & $<.001$ & -33 & -41 & -16 & 20 \\
\hline 17 & L InferiorTemporal Gyrus & 15.19 & $<.001$ & -56 & -9 & -16 & 21 \\
\hline 18 & L Sub Gyral & 15.58 & $<.001$ & -39 & -12 & -8 & 21 \\
\hline 19 & L SuperiorTemporal Gyrus & 20.46 & $<.001$ & -48 & 6 & -3 & 22,38 \\
\hline 20 & R MiddleTemporal Gyrus & 4.67 & .003 & 59 & -60 & 11 & 37,39 \\
\hline 21 & R SuperiorTemporal Gyrus & 7.05 & $<.001$ & 64 & -40 & 21 & $0,0,13,22,42$ \\
\hline \multicolumn{8}{|c|}{ Limbic lobes } \\
\hline 22 & R CingulateGyrus & 15.48 & $<.001$ & 16 & -27 & 39 & $24,31,32,9$ \\
\hline \multicolumn{8}{|c|}{ Deep gray (Sub lobar areas) } \\
\hline 23 & L CaudateCaudate Head & 2.46 & .028 & -11 & 15 & -6 & \\
\hline 24 & L Insula & 27.92 & $<.001$ & -52 & -34 & 19 & 13 \\
\hline 25 & L LentiformNucleusMedialGlobus Pallidus & 13.90 & $<.001$ & -15 & -4 & -3 & \\
\hline 26 & L LentiformNucleusPutamen & 3.49 & .009 & -19 & 12 & -7 & \\
\hline 27 & R Insula & 17.34 & $<.001$ & 42 & 12 & 13 & 13 \\
\hline \multicolumn{8}{|c|}{ Additional regions } \\
\hline 28 & L Anterior LobeCulmen & 14.93 & $<.001$ & -24 & -40 & -17 & \\
\hline 29 & R Anterior LobeCulmen & 16.67 & $<.001$ & 12 & -60 & -10 & \\
\hline 30 & R Anterior Lobe & 3.19 & .012 & 11 & -42 & -27 & \\
\hline 31 & R Posterior LobeCerebellarTonsil & 3.93 & .006 & 6 & -47 & -33 & \\
\hline \multirow[t]{2}{*}{32} & R Posterior LobeDeclive & 22.13 & $<.001$ & 12 & -62 & -11 & \\
\hline & Positive difference & & & & & & \\
\hline \multicolumn{8}{|c|}{ Limbic lobes } \\
\hline 1 & Right Limbic LobeAnteriorCingulate GM & 4.23 & .004 & 5 & 19 & 19 & 6,33 \\
\hline \multicolumn{8}{|c|}{ Additional regions } \\
\hline 2 & Left CaudateGMCaudate Body & 7.89 & $<.001$ & -9 & 17 & 13 & 2 \\
\hline
\end{tabular}

Anatomical labels and associated $T$ statistical values are listed. $T$ scores from the omnibus analyses of 6 participants for each ROI are presented 


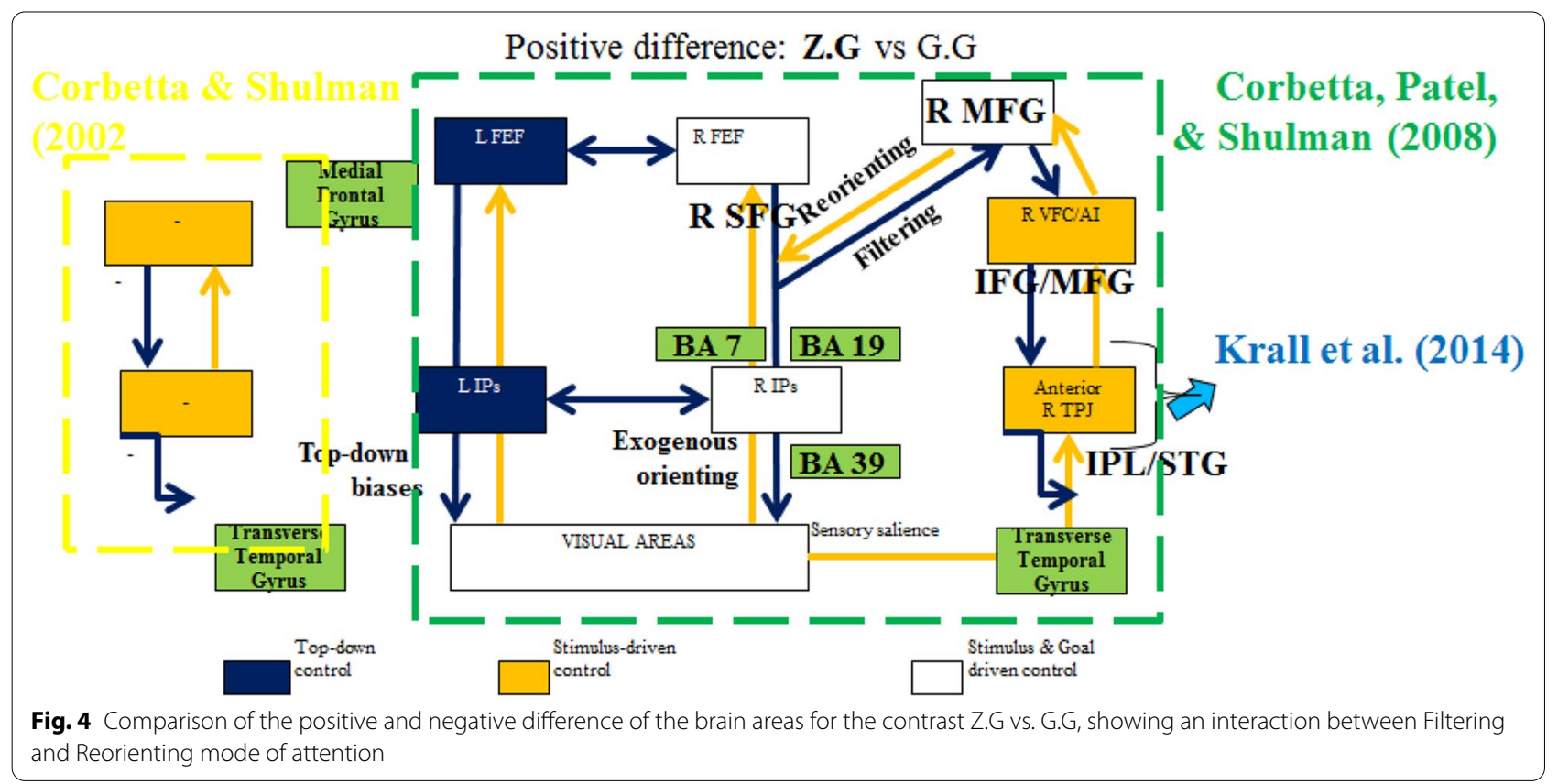

SFG with BA 6 and the Right Cingulate Gyrus with BA 24 are activated with both positive and negative contrast (see the highlighted results in Table 7). In addition, the left Precentral Gyrus is activated differently in this contrast, which informs different motor response than the other contrasts. Again, there are frontal differences in left and right MFG (up to $46 \mathrm{~mm}$ and $44 \mathrm{~mm}$, respectively). Results showed that the greatest differences measured occurred towards the most frontal area of the brain, with the greatest frontal differences measuring up to $50 \mathrm{~mm}$ in the left SFG and up to $56 \mathrm{~mm}$ in the right SFG, having the more frontal activation in the right hemisphere. Overall these differences in the Prefrontal Cortex by the trial before the $\mathrm{G}$ condition in analysis are supporting hypothesis $\mathrm{H} 2$ and suggest the more frontal activation for the switching from simultaneous Novel and Goal to the Goal which is also concordant with Koechlin's model (2003) of the frontal episodic attention control and with Corbetta's model [20] lateralizing to the right hemisphere. Figure 4 on the bottom shows this contrast. Our results also extended the idea on steady-state visual evoked potentials (SSVEP) where frontal electrodes in 2-oddball attention tasks were found responsible for suppression of distractor responses [24], i.e. how different 2-oddball task maybe seen locally in prior context in the present 4-oddball task experiment.

Table 4 also shows the frontal differences in the left and right Anterior Cingulate Cortex (ACC, up to $34 \mathrm{~mm}$ and $30 \mathrm{~mm}$, respectively), this is consistent with the view that ACC is involved in conflict monitoring (reviewed by van
Veen and Carter [25]) which is the previous context in our analysis.

In Table 5, the contrast of sequences N.G and Z.G is shown. Both hemispheres in occipital and limbic brain areas showed differences strongly biased to the Z.G contextual condition and both hemispheres showed activation for frontal, temporal and parietal in positive and negative contrasts. According to the results, the Left MedialFrontal Gyrus, Left SFG, Right MedialFrontal Gyrus, Right MFG, Right Precentral Gyrus, Right SFG, Left MiddleTemporal Gyrus and Right STG with different BAs are activated with both positive and negative contrast (see the highlighted results in Table 5). Also, Table 5 showed differences in several frontal regions biased to the N.G condition. Again, there are frontal differences in the left and right MFG (up to $46 \mathrm{~mm}$ and $44 \mathrm{~mm}$, respectively). Results showed that the greatest differences measured occurred towards the most frontal area of the brain, with the greatest frontal differences measuring up to $50 \mathrm{~mm}$ in the left SFG and up to $56 \mathrm{~mm}$ in the right SFG, having more frontal activation in the right hemisphere. Overall these differences in the Prefrontal Cortex by the trial before the $\mathrm{G}$ condition in analysis support hypothesis $\mathrm{H} 2$ and suggest more frontal activation for the switching from simultaneous Novel and Goal to the Goal which is also concordant with Koechlin's model of the frontal episodic attention control and with Corbetta's model lateralizing to the right hemisphere [20].

In Table 4, there are also differences in the left and right Anterior Cingulate Cortex (ACC). This is consistent with 


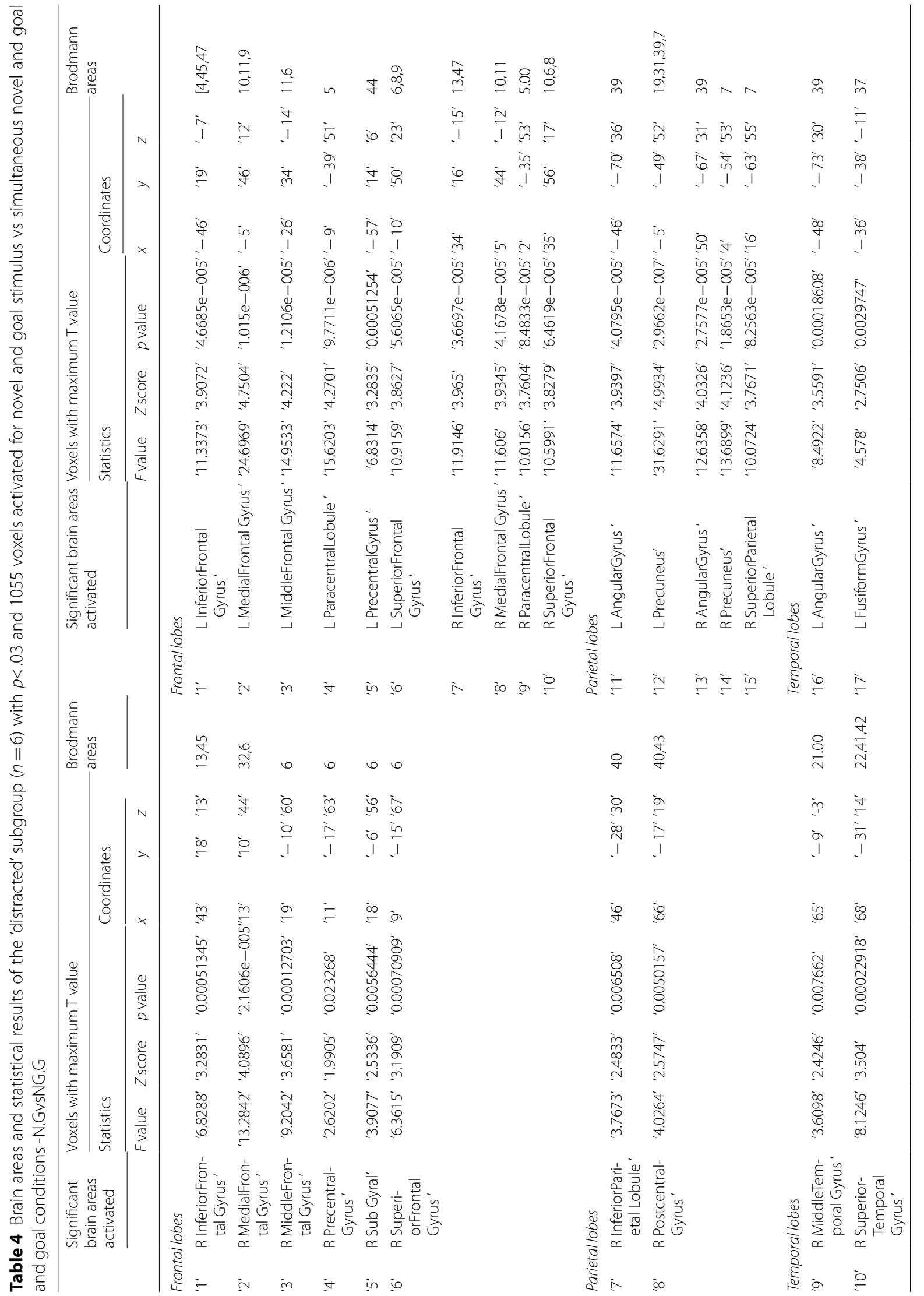




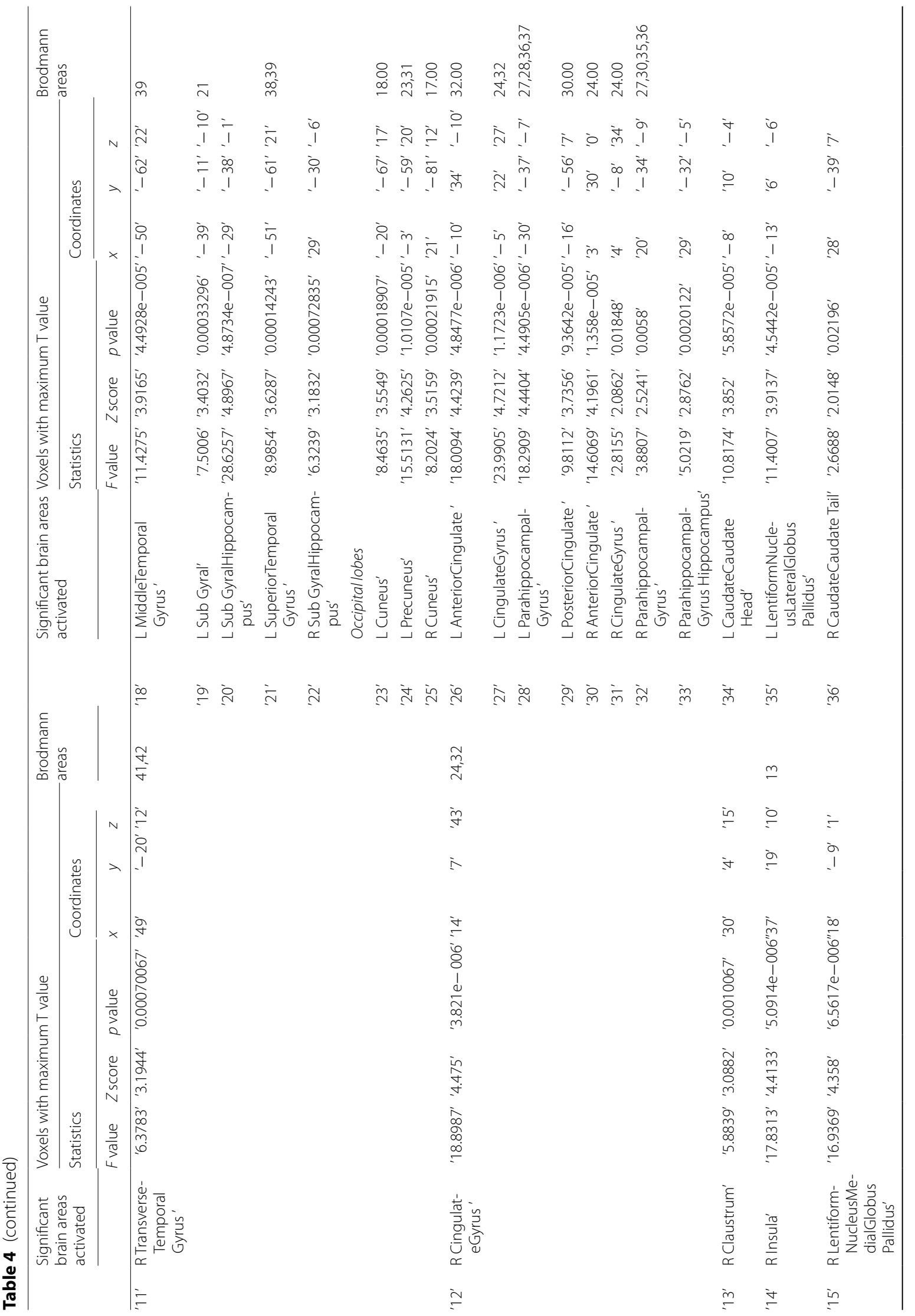




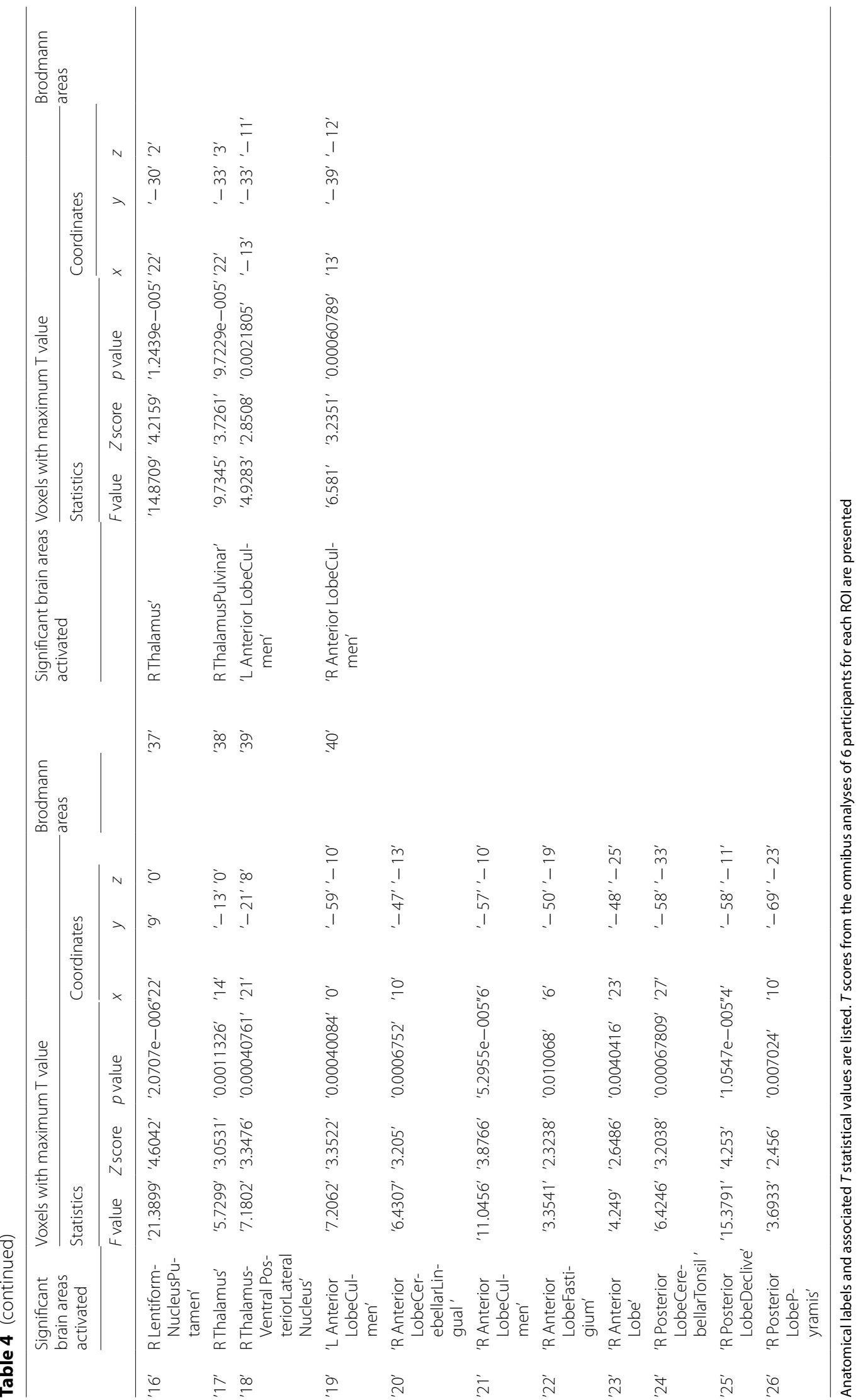


the view of ACC in conflict monitoring [25] which is the previous context in our analysis.

Table 6 shows the contrast of sequences N.G and Z.G. Both hemispheres in parietal brain areas showed differences strongly biased to the Z.G contextual condition and both hemispheres showed activation for frontal, temporal, occipital and limbic in positive and negative contrasts. According to the results, the Right SuperiorTemporal Gyrus with the BA 22 with both positive and negative contrast (see the highlighted results in Table 6). Further, Table 6 showed frontal differences in two frontal regions biased to the N.G condition. In these contrasts, there are frontal differences in right MFG biased on N.G (up to $37 \mathrm{~mm}$ ). The other great frontal difference is up to $32 \mathrm{~mm}$ in the right IFG. Therefore, the more frontal activation occurs in the left hemisphere. Overall, these differences in the Prefrontal Cortex by the trial before the G condition in analysis support hypothesis $\mathrm{H} 2$ and suggest more frontal activation for the switching from Novel to the Goal which is also concordant with Koechlin's model of the frontal context attention control [5] and with Corbetta's model lateralizing to the right hemisphere [20].

Table 6 also shows the differences in the left and right Anterior Cingulate Cortex (ACC). This is consistent with the view of ACC in conflict monitoring [25], which is the previous context in our analysis.

\section{Discussion for contrasts, context to extend multimodal task}

The first results discussed here focus on the 6 'distracted' participant's analysis which showed more significant brain activations than found for the whole group of 11 participants.

The analysis of these fMRI data (a) explored the effect of prior context across participants supporting $\mathrm{H} 2$ but only for 'distracted' participants; (b) explored novel response generators and simultaneous novel and target response generators relative to the standard goal condition supporting $\mathrm{H} 1$ but only for 'distracted' participants; (c) resulted in a larger recruiting neural response at the prefrontal cortex having less areas for standard goal stimulus and standard previous motor response and (d) attempted to find a possible explanation for the observed smaller than expected Novel sound ERP amplitudes. Last two analyses allowed having a grasp for modelling of auditory and motor function of the human brain (H3).

\subsection{RT results suggest that the novelty effect may vary between causing alerting and orienting}

The RTs observed in the orienting subgroup were slower (20 to $70 \mathrm{~ms}$ ) in the simultaneous novel and target (NG) condition suggesting that the focus of attention can be shifted with the introduction of a novel stimulus alongside the target in the mental representation of the auditory scene. In the literature we find this range of reaction times in orienting to alerting stimuli by Fan and colleagues [26]. According to Fan and colleagues, behavioural reaction time differences in alerting would be around $60 \mathrm{~ms}$, orienting around $31 \mathrm{~ms}$ and conflict monitoring around $102 \mathrm{~ms}$ [26]. Brain areas of specific interest in the number parity decision task.

In the case of the parietal lobes: in the $\mathrm{Z}$ vs. $\mathrm{G}$ contrast the Right Precuneus were similarly activated only in this contrast; in the NG vs. G contrast the L/R Angular Gyrus, L/R Inferior Parietal Lobule and Left Superior Parietal Lobule (SPL) showed different activations only in this contrast for F-value difference; and in the $\mathrm{N}$ vs. G contrast the Left Precuneus showed similar activations only in this contrast while in the motor cortex the Right Paracentral Lobule showed different activations only in this contrast. Therefore, in the NG vs. G contrast, IPL and SPL showed different activations. Activation in the Precuneus ( $p \leq 0.0005$ uncorrected) is of interest because Precuneus is associated with reaching activity $[27,28]$. Although in the present experiment the hand is not reaching different places, the selected finger (index or middle) is reaching the button for the task, the Goal and Novel stimulus showed an activation similar to the tendency to reach the novel, with different brain activations suppressing the button press in N vs. G more in the right Precuneus and allowing the button press in NG vs. G and $Z$ vs. G in left and right Precuneus. Taking altogether the results for the contrast NG vs. G there is consistent with recent subdural electrodes in humans in the IPS, SPL and Precuneus for reaching a cup from a resting position [29].

On the temporal lobes: in the $\mathrm{Z}$ vs. G contrast the Left Sub Gyral area showed similar activations only in this contrast while in the different contrasts the L/R Transverse Temporal Gyrus (TTG) showed different activations. This is consistent with the result of the $750 \mathrm{~Hz}$ tone which activated more voxels in the medial area of the TTG, whereas the $2000-\mathrm{Hz}$ tone activated more voxels in the lateral TTG [30]. Moreover, the Right Superior Temporal Gyrus (STG) has different activations in the different contrasts, which has been reported to be activated more by speech and frequency modulated tones [31]; in the NG vs. G contrast the L/R Angular Gyrus, Left Fusiform Gyrus, L/R Sub Gyral Hippocampus and Right Middle Temporal Gyrus showed different activations only in this contrast. Hippocampus and the different prefrontal areas activated during the task according to the presence of NG appeared by the presence of the novel when there is not an explicit sequence and having several conditions, in spite of Savalia and colleagues findings [9]. 


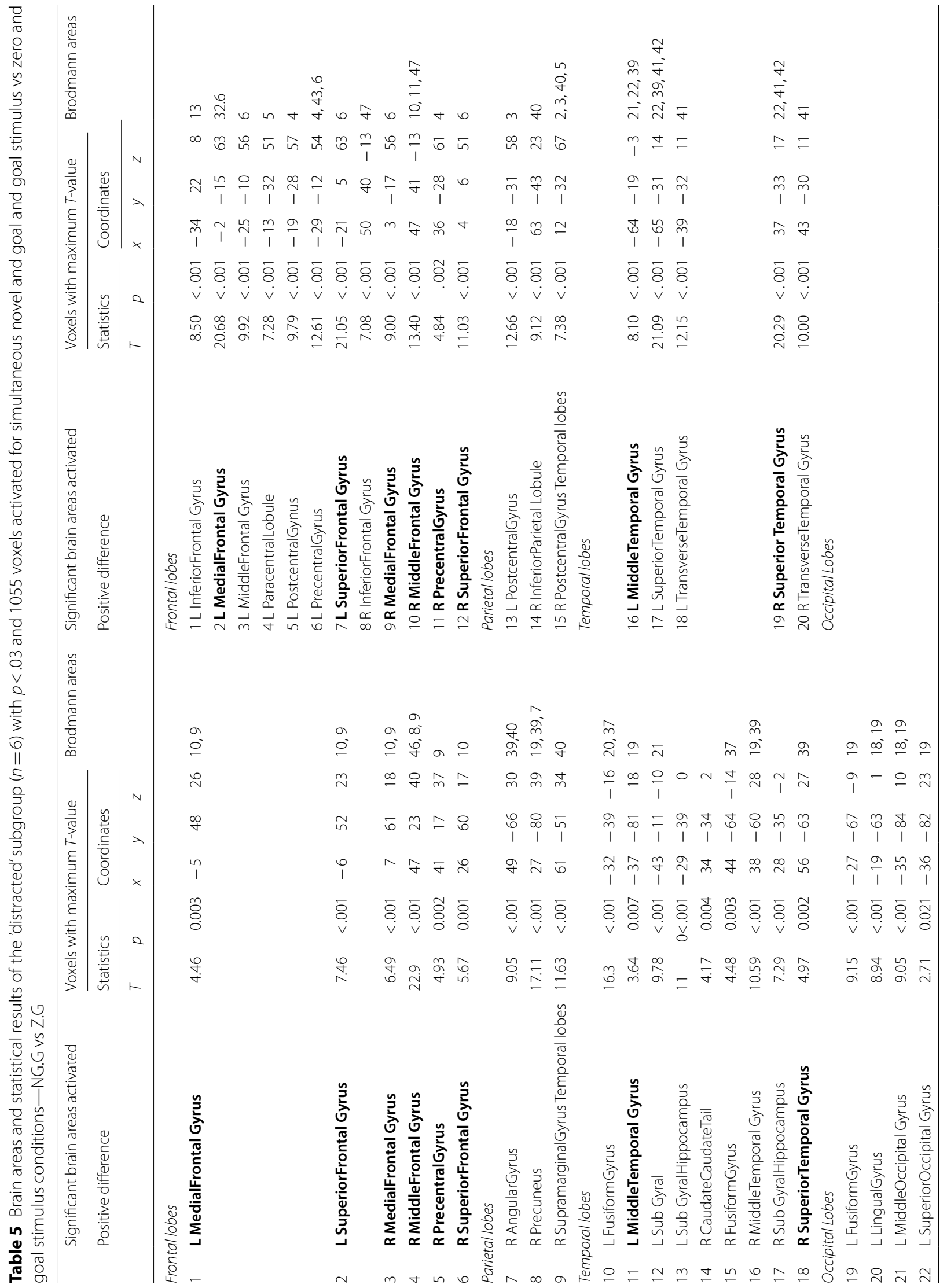




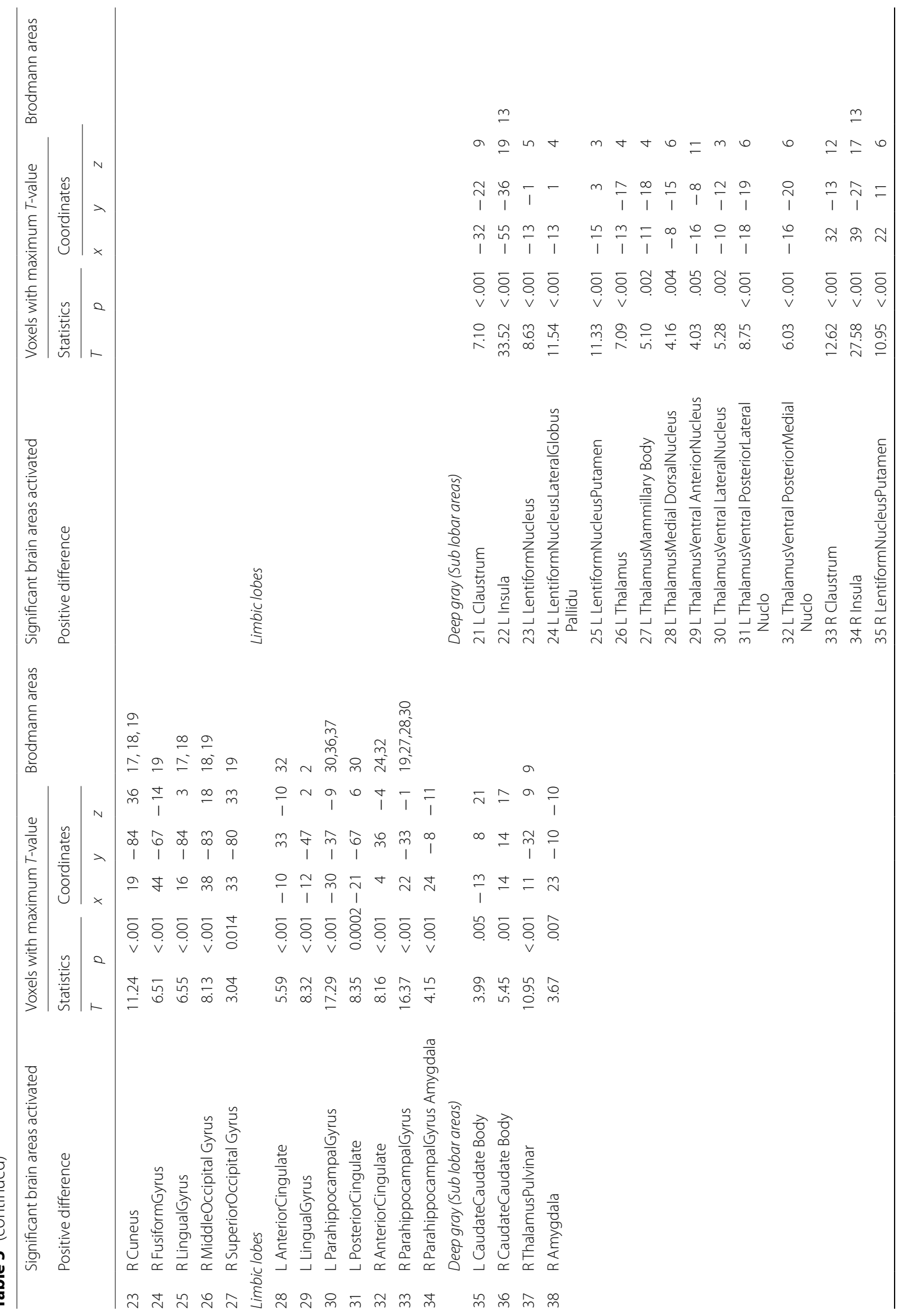




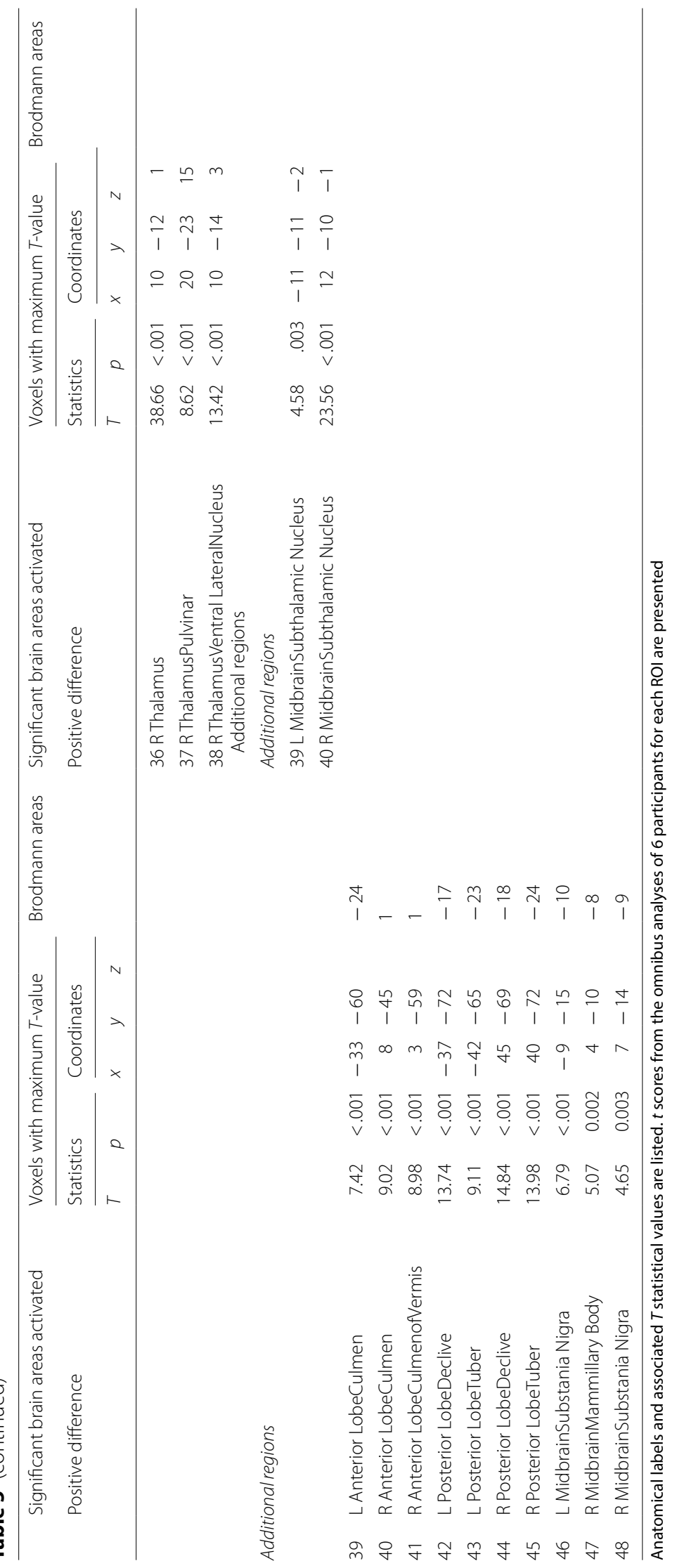




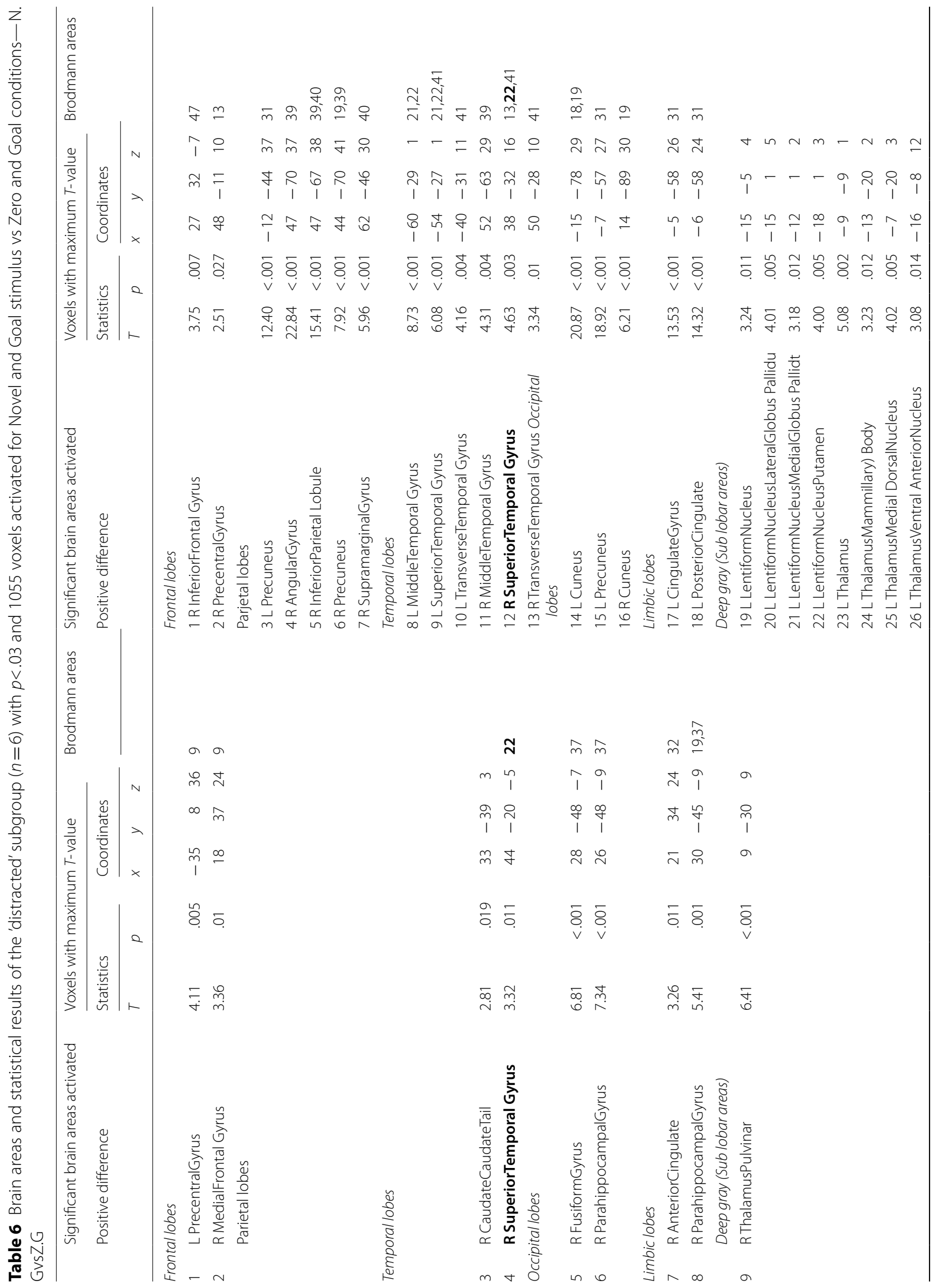




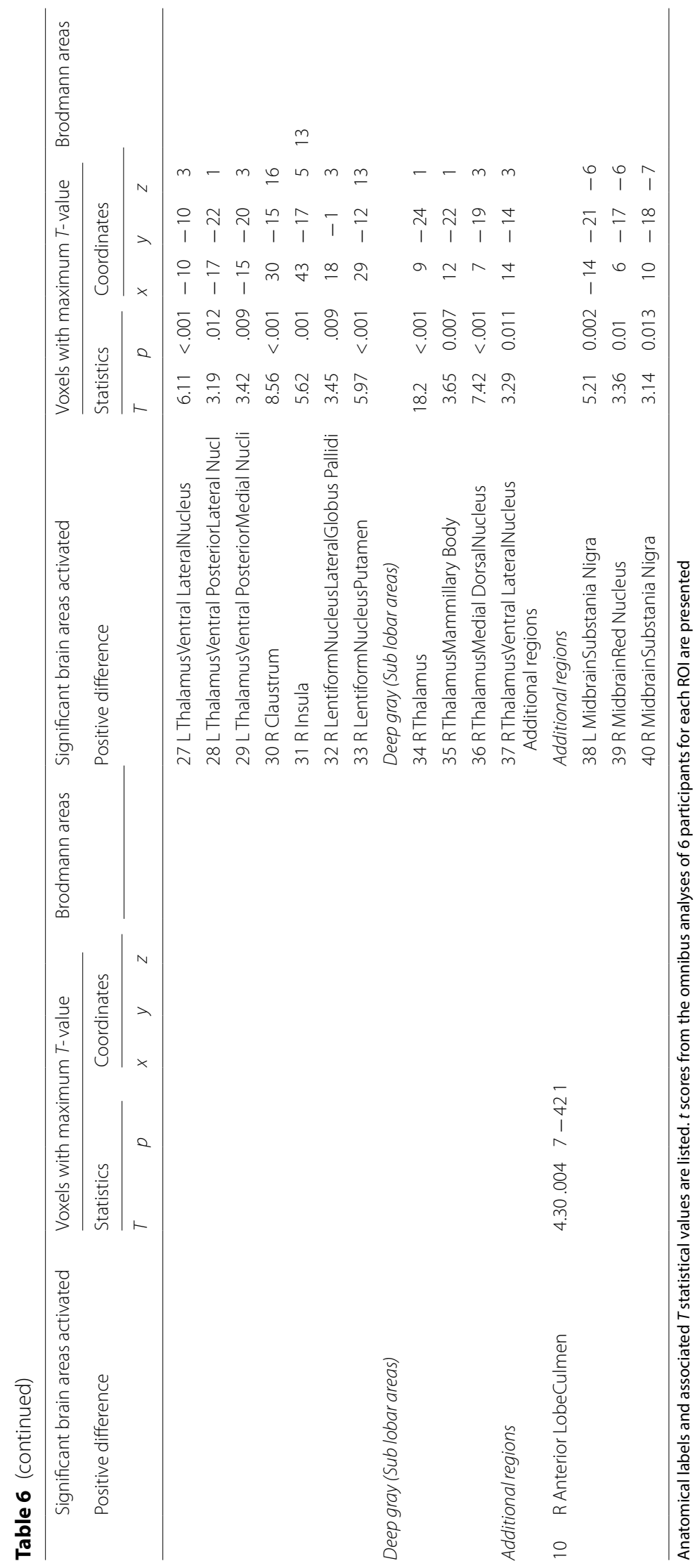


In the case of the occipital lobes: in the $\mathrm{Z}$ vs. $\mathrm{G}$ contrast the Right Fusiform Gyrus showed different activations only in this contrast; in the NG vs. G contrast the Right Cuneus/Precuneus Right Lingual Gyrus and Right Superior Occipital Gyrus showed different activations only in this contrast; and in the $\mathrm{N}$ vs. $\mathrm{G}$ contrast the Left Cuneus/Precuneus showed similar activations only in this contrast. FusiformGyrus activation reduces with repeated presentations, also when the performance of the participant is better [32]. In the present results, the L FusiformGyrus is more activated in the Novel than the $\mathrm{Z}$ and NG conditions, having clear differences at Goal as an object identification. However, there is no clear difference in the contrast of different conditions $\mathrm{N}$ vs $\mathrm{G}$ and N.G vs G.G. This supports the view that the orienting response is sensitive to the degree of familiarity with the experiment [33].

\subsection{Prefrontal cortex and motor responses in the preceding trial $(\mathrm{H} 2)$}

Results showed that the Precentral Gyrus (PrG) motor area was activated differently in $Z$ vs. G, N vs. G and NG vs. $G$ contrasts. Activations were more ventral with relatively greater activations for the $\mathrm{N}$ condition (BA 43), and with relatively greater activations in different BAs in the NG vs. G contrast, in the left BA 6 for the NG condition and right BAs 4, 6 and 44 with relatively greater activations for the $G$ condition. Moreover, taking into account the contextual contrasts, activations for Z.G vs. G.G contrast produced larger activation in the Right PrG (BAs 4 and 6) and for the N.G vs. G.G contrast had relatively greater activations for the N.G condition on the Left PrG (BA 4) and Right PrG (BA 4, 44 and 6). Therefore, overall all these results different prefrontal control is seen at PrG.

Although motor response is usually activated in the contralateral side, in this experiment the right hand was used in the parity decision task whilst some ipsilateral responses in the Left PrG were activated for N.G condition over G.G condition. Considering the change of the fundamental frequencies between N and G conditions, this left ipsilateral result to the right hand of response is consistent with frequency changes greater than $30 \mathrm{~Hz}$ observed for harmonic tones [34]. Thus, the Novel before a Goal makes more contribution to different motor area activations and similar activations than the NG conditions. Therefore, the 'distracted' participants showed a stronger attention to the task than to the motor control in $\mathrm{N}$ vs. $\mathrm{G}$ contrast and the motor control switch between N.G and G.G conditions, which is similar to the conflict motor control switch between NG and G conditions. Therefore, the motor response may be used in explaining the prefrontal control in the light of $\mathrm{H} 2$. This part of the discussion is expanded in the next part of the discussion which studies context from the point of view of the previous trial.

\subsection{Prefrontal cortex and context given by the immediately previous trial $(\mathrm{H} 2)$}

Tables 3 and 4 show that there are more differences in NG.G vs. N.G than in G.G vs. N.G, consisting of more frontal areas and towards to the front as well for NG.G vs. N.G, which is consistent with the different frontal activations in the contextual approach of the hypothesis $\mathrm{H} 2$.

More insights derived from the results driven by hypothesis $\mathrm{H} 2$ are analysed in Table 7 . This shows the comparison of the five contrasts analysed (first column). From Z.G vs. G.G to N.G vs. Z.G contrasts, it looks like the effect of a previous Novel stimulus is to increase the activation of the prefrontal areas. When both contrasts are compared to the N.G vs. G.G contrast, this increased activation of additional prefrontal areas is corroborated, and also the change of motor response results analysed in the previous section in the activation of additional prefrontal areas. In Table 7, when the first and third rows are compared with the fourth and fifth row, respectively, a similar increase of the number of areas in the prefrontal region is shown. Result suggested, in Table 7, when instead of G is NG part of the increased number of PFC areas is because of the recruiting of the brain areas closer to the ACC.

ACC activation was shown in both hemispheres (see Tables 7 and 8) related to NG.G (versus N.G and Z.G) and in the left hemisphere (see Table 3) related to N.G (versus G.G). First, this ACC activation is consistent with the view that the ACC facilitates control of attention [25]. These results showed consistency with conflict monitoring being more frontal and deeper for NG.G vs. N.G contrast, see Left ACC at $(-10,34,-10) \mathrm{mm}$ and the Right $\mathrm{ACC}$ at $(3,30,0) \mathrm{mm}$ in Table 7). Alongside the comparison in Table 7, these results in frontal areas are not only consistent with the prefrontal control proposed by Koechlin and colleagues [4], but the R SMG is also consistent with the model of control of attention proposed by Corbetta and colleagues [7].

\section{4 fMRI for 'distracted' participants showed left and right brain areas for contextual conditions in the attention model ( $\mathrm{H} 1$ and $\mathrm{H} 2)$}

First, the results of the Z.G vs. G.G contrast showed different right parietal activation and no different occipital areas as the signature of this contrast. The results are summarized in the graphic in Fig. 4 and they have shown consistency with the visual stimulus-driven 
attention network model of Corbetta and Shulman [7] as shown for the left hemisphere in the dotted rectangle in yellow. Although the positive contrast results are not exactly consistent with the reorienting of attention of Corbetta and colleagues [20], the activations in Brodmann Areas 7, 19 and 39 may be related to activity in the R IPs. However, the FEF is not clearly activated. In addition, the negative contrast only showed significant activation of the left Medial Frontal Gyrus without a clear different activation of the control of attention for the G.G condition. Of course, this can be explained because the current trial $(G)$ has mostly the same properties of the frequently previous trial type $(G)$. These interpretations suggest that the Z.G is evoking an interaction of the stimulus and goal-driven network differently to the pattern orienting of attention, while the IPs is suggested to be related to BAs 7, 19 and 39 (see dotted rectangle in green).

Second, when the N.G and G.G contextual conditions are more involved in a different frontal control of attention: the results of the N.G vs. G.G contrast showed different left and right parietal activation and no differences in occipital areas as the signature of this contrast. The results are summarized in the graphic in Fig. 5. The results support right and left (see dotted rectangle in yellow) hemispheres in the stimulus-driven attention network of Corbetta and Shulman [7] suggesting the control of attention in the N.G sequence. Although, the positive contrast results are not exactly consistent with the reorienting of attention of Corbetta and colleagues [20], but the Brodmann Areas 7, 40 and 39 may be enclosing the activity in the R IPs. Further, the negative contrast only did not show significant activation of the cortex; again, this can be explained because the current trial (G) has mostly the same properties of the previous trial $(G)$. These interpretations suggest that the N.G is evoking an interaction of the stimulus and goal-driven network similar to the pattern orienting of attention (see dotted rectangle in green).

Finally, right lateralized Thalamus in the Pulvinar has shown significant response at novel response (Tables 5 , 7,8 ) also bearing the prior stimulus (Tables 11,12 ) as well as no motor response (Table 6) or previous motor response (Table 7). This result is concordant with the finding of different management on Pulvinar on selective attention [35], here under different conditions modulation was found according to different prefrontal areas. Moreover, this modulation would be concordant with the finding that the ventrolateral Pulvinar receives inputs from the prefrontal cortex, helping in modulation of visual processing and attention [36]. Although our methods in the current study are not sensitive enough to examine ventrolateral Pulvinar, we have found that it can encode prior context either auditory signals or motor responses that can be explore to study lateral geniculate nucleus (LGN) to top-cortical areas and from these areas to Pulvinar-like structures [37].

\section{5 fMRI and ERP comparison and the anterior cingulate cortex}

Comparing fMRI and ERP results in the 'distracted' subgroup: (a) the Anterior Cingulate Cortex (ACC) is not activated differently between $\mathrm{Z}$ and $\mathrm{G}$ conditions (Table 2) and the ERP deflection around $200 \mathrm{~ms}$, biased for $\mathrm{Z}$ condition negatively to the left frontal electrode $\mathrm{F} 7$ and positively to the right frontal electrode F8 in Additional file 1: Figure S1; (b) Right ACC is activated differently between NG and G conditions (Additional file 1: Table S4) being more frontal for NG condition in the right ACC (BA 32) and more posterior for the G condition (BA 32 ) and the negative ERP deflection around $200 \mathrm{~ms}$ in the right electrode F8 (in Additional file 1: Figure S1) and stronger Left ACC is activated differently between NG

Table 7 Input/Output comparison of the number of Brain areas for the different contrasts explored

\begin{tabular}{|c|c|c|c|c|c|c|c|c|c|}
\hline \multirow[t]{3}{*}{ Contrasts } & \multirow{3}{*}{$\begin{array}{l}\text { Previous input } \\
\text { Previous stimulus }\end{array}$} & \multirow{3}{*}{$\begin{array}{l}\text { Previous output } \\
\text { Previous Motor } \\
\text { response }\end{array}$} & \multicolumn{6}{|l|}{ Current output } & \multirow[t]{3}{*}{ Table reported } \\
\hline & & & \multirow[t]{2}{*}{$\begin{array}{l}\text { Number of frontal } \\
\text { areas activated }\end{array}$} & \multicolumn{2}{|c|}{$\begin{array}{l}\text { Max frontal } \\
\text { axis (mm) }\end{array}$} & \multirow[t]{2}{*}{$\begin{array}{l}\text { Number of frontal } \\
\text { areas activated }\end{array}$} & \multicolumn{2}{|c|}{$\begin{array}{l}\text { Max frontal } \\
\text { axis (mm) }\end{array}$} & \\
\hline & & & & Left & Right & & Left & Right & \\
\hline Z.G vs G.G & Number vs number & not vs do & 9 & -24 & 28 & 1 & 39 & - & Table 2 \\
\hline N.G vs Z.G & Novel vs number & not vs not & 2 & 8 & 37 & 2 & - & 32 & Table 6 \\
\hline N.G vs G.G & Novel vs number & not vs do & 9 & 41 & 37 & - & - & - & Table 3 \\
\hline NG.G vs Z.G & $\begin{array}{l}\text { Novel }+ \text { number vs } \\
\text { number }\end{array}$ & do vs not & 6 & 52 & 61 & 12 & 22 & 41 & Table 5 \\
\hline NG.G vs N.G & $\begin{array}{l}\text { Novel + number vs } \\
\text { novel }\end{array}$ & do vs not & 6 & & 18 & 10 & 50 & 56 & Table 4 \\
\hline
\end{tabular}


and G conditions (Additional file 1: Table S4) being with relatively greater for the NG condition in the left ACC (BA 32) and the negative ERP deflection around $200 \mathrm{~ms}$ is stronger to the left frontal electrode F7 (in Additional file 1: Figure S1); (c) difference between N and G conditions (Additional file 1: Table S1) and no clear difference around the ERP at $200 \mathrm{~ms}$ (F7 and F8 in Additional file 1: Figure $\mathrm{S} 1$ ). These results suggest that $\mathrm{ACC}$ is linked to N200 for NG condition in both hemispheres. On the other hand, in the $\mathrm{N}$ vs. G contrast positive and negative activation differences in ACC were observed and no clear ERP different deflections around $200 \mathrm{~ms}$, namely MisMatch Negativity. This analysis is consistent with the view of N200 and ACC in conflict monitoring studies [25]. However, but, because of MMN, it is not clear about the Novel effect.

Moreover, ACC activation was shown to be different across the other contextual contrasts (Z.G vs. G.G, N.G vs. G.G, N.G vs. NG.G, NG.G vs. Z.G and N.G vs. Z.G) and the relatively greater activation was shown not only for novel, but also for Zero condition. Therefore, ACC relative activations were sensible to contextual changes depending on Goal (G), Non-Goal (Z and N) and Novel ( $\mathrm{N}$ and NG) signals.

In the 'distracted' participants, the contrast between NG.G and N.G was evaluated for the ACC. Results showed relatively greater activation for the N.G condition in the Left (BA 32) and Right (BA 24) ACC. This suggests that ACC produces different activations depending on the previous context for stimulus-driven network and the conflict monitoring effect. When the contrast between NG.G and N.G conditions in 'all the participants' was evaluated, there were no significant differences in ACC activation and this suggests that ACC in the alerting state does not produce different activations for the different Novel trials presented before the current Goal trial. These differences between the 'distracted' and the 'all participants' would explain the difference of the analysis of the ERP at N200 in Potter's study [38] and ACC in fMRI in the present analysis of the 'distracted' subgroup.

Another possible comparison would be a further eye field activation in FMRI and beta waves in EEG such as was found for higher arousal levels [39]. The present analysis may accommodate the role of the FEF in attention when the Corbetta's model of attention is considered. Therefore, a further limitation in the present analysis is that this was the third task in the participants and possibly the results for FEF in the 'distracted' participants added to the inhibition of return for $\mathrm{Z}$ vs $\mathrm{G}$ contrast were related with the arousal level to keep the answer to the task in the auditory attention task.

In practical use to add in this discussion, this experimental discussion may have a theoretical extension to be used by BCI systems that involve the management of neural network and learning systems architectures. This was addressed in the following conclusion.

Limitations of 12 participants were compensated by a FDR analysis (similar number in fMRI statistic comparison by Nichols [40]) and bearing in mind current theory of attention and a similar auditory paradigm, which

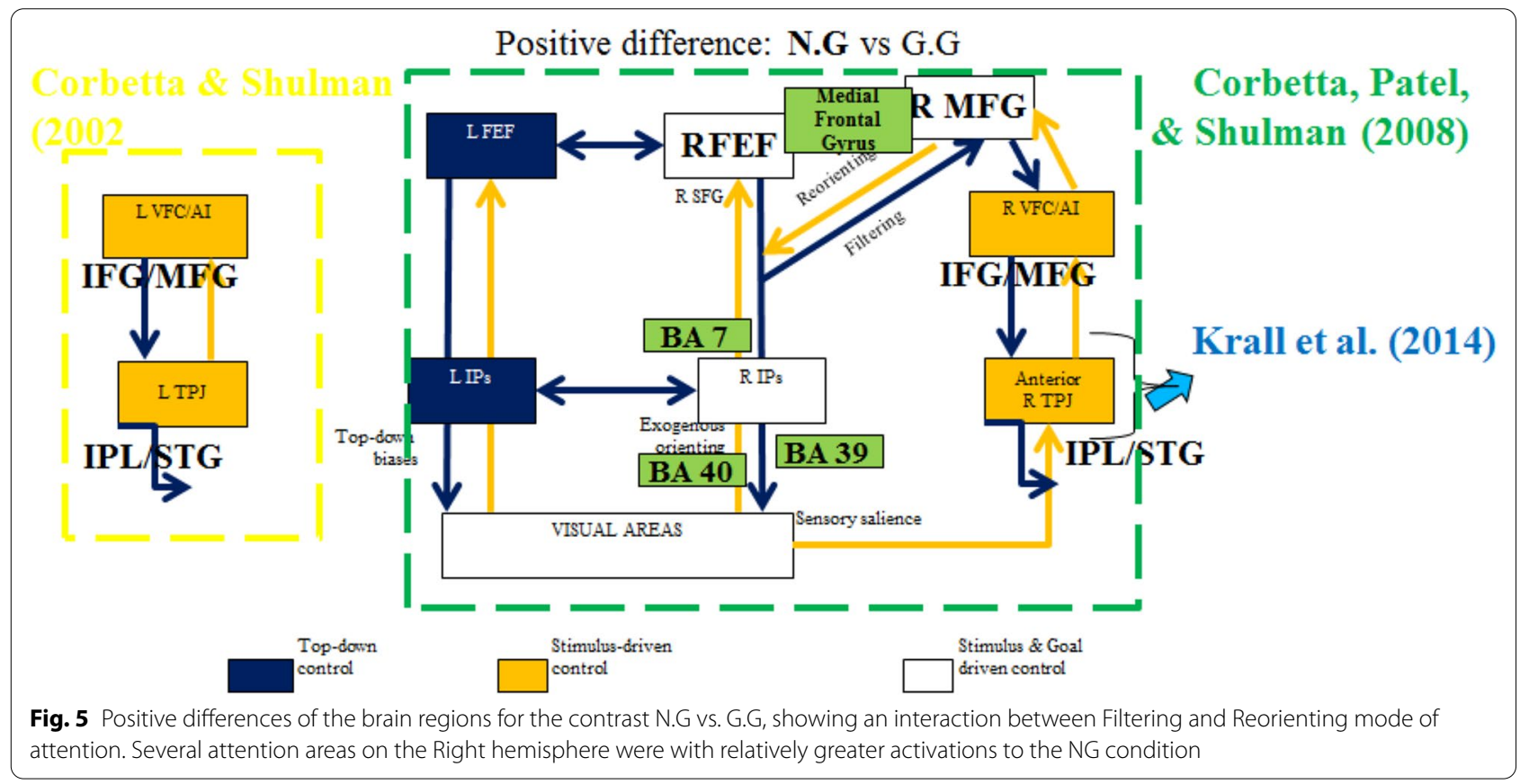


explored context with EEG in schizophrenic participants [3].

\section{Conclusion: improvement of modelling novel response due to previous motor response}

Given results and discussion, the sequence of stimulus studied has shown different activation of the hippocampus areas which have been in favour of the theory or cortical and subcortical loop for sequence proposed by Savalia and colleagues [9]. Moreover, the present results have reported when a sequence is interrupted by a novel (simultaneously) the subcortical loop with the hippocampus is also activated. This has extended Mugruza-Vassallo and Potter studies of temporal stimulus sequence with EEG [3] to fMRI brain regions and following their analysis and extension of management of novel stimulus modulated by the previous motor answer a model is proposed in Fig. 6 solving part of the puzzle proposed by Livnet and Zador [14]. These consistencies make it of interest to explore another experiment to study the EEG results in more detail and combine with the fMRI analysis to seek for the explanation of these partial consistencies.

Bearing in mind eye movement research in response to an auditory experiment has shown results in pupil dilation response [41], the present findings on motor modulation of attentional processing would be extended by a broader motor response. Moreover, the model would modify the Information Dynamics of Thinking (IDyOT) model for language and music of Forth and colleagues [42] may bear in mind previous motor response and unexpected external stimulus. Forth and colleagues proposed a mechanism for predicting when a perceptual event will happen, given an existing sequence of past events, which may be musical or linguistic [42].

Evolutionary multitasking computation [19] maybe best based on multi-objective optimization of cortical prefrontal cortex for different incoming stimulus employing stimulus features for objective functions (f) for vectors of decision variables $(\mathrm{y})$ in the search space $(\mathrm{Y})$ following Eq. 3, considering 4 conditions:

$$
\operatorname{maximize}(y \in Y) f(y)=\left|f_{1}(y) ; f_{2}(y) ; f_{3}(y) ; f_{4}(y)\right| \text {. }
$$

Then for $K=4$ different tasks (T1, T2, T3, T4) the MOP in terms of the populations would follow Eq. 3, but bearing in mind the different responses due to previous motor command. In this way $f_{k}(y)$ will depend on the neural processing of previous motor response $y\left(m_{(n T-T)}\right)$ and the current motor response $y\left(m_{(n T)}\right)$, as seen in (4):

$$
f_{k}(y)=g_{k, 1}(y(s)) g_{k, 2}\left(y\left(m_{(n T)}, y\left(m_{(n T-T)}\right)\right)\right) .
$$

Also bearing in mind our "inhibition of return" results, they influence on the number of prefrontal areas modulated. Therefore, an additional input would be needed to maximize decision variables going for at least $m=\{0,1\}$, 0 for no motor response and 1 for motor response in (5). This would be valid for 2-oddball tasks (e.g. [24]):

$$
\begin{aligned}
& \max (y \in Y) \sum_{k=1}^{4} \int z f_{k}(z) \cdot\left[\sum_{j=1}^{4} w_{j k, m(k-1)} \cdot p_{j, m(k-1)}(z)\right] \cdot \mathrm{d} z . \\
& \left\{w_{j k} \cdot p_{j}(z)\right\}
\end{aligned}
$$

Therefore, we may have $f_{1}(y)$ relying on $\mathrm{G}$ condition, as well as $f 1(y), f 2(y), f 3(y), f 4(y)$ relying on G.G, Z.G, N.G and NG.G from Eqs. 6 and 7. The power of analysis (7.a, 7.b, 7.c, 7.d) is better than for only one condition (6.a). In (6) prior context would not be reached by almost any $g g_{i, j}$ in particular:

$$
\begin{array}{r}
f f 1(y)=g g_{1,1}(y(s=G)) g g_{1,2}(y(m(n T), y(m(n T-T)))), \\
(6 . \mathrm{a}) \\
f f_{2}(y)=g g_{1,2}(y(s=Z)) g g_{k, 2}\left(y\left(m_{(n T)}, y\left(m_{(n T-T)}\right)\right)\right), \\
(6 . \mathrm{b}) \\
f f_{3}(y)=g g_{1,3}(y(s=N)) g g_{k, 2}\left(y\left(m_{(n T)}, y\left(m_{(n T-T)}\right)\right)\right), \\
f 6 . \mathrm{c}) \\
f f_{4}(y)=g g_{1,4}(y(s=N G)) g g_{k, 2}\left(y\left(m_{(n T)}, y\left(m_{(n T-T)}\right)\right)\right) .
\end{array}
$$

An example of the power of analysis by $f f$, we may have Z.G vs G.G and on other hand N.G vs NG.G where the Pulvinar was activated as well employing different parts of Eq. (7), where prior context may be considered by $g g_{i, j}$ in particular can account prior context contrasts, being different from inhibition of return, standard stimulus and both different way of novel stimulus:

$$
\begin{array}{r}
f 1(y)=g_{1,1}(y(s=G . G)) g_{1,2}(y(m(n T), y(m(n T-T)))), \\
(7 . a) \\
f 2(y)=g_{2,1}(y(s=Z . G)) g_{2,2}(y(m(n T), y(m(n T-T)))), \\
(7 . b) \\
f 3(y)=g_{3,1}(y(s=N . G)) g_{3,2}(y(m(n T), y(m(n T-T)))), \\
(7 . \mathrm{c}) \\
f 4(y)=g_{4,1}(y(s=N G . G)) g_{4,2}(y(m(n T), y(m(n T-T)))), \\
(7 . \mathrm{d}) \\
f_{5}(y)=g_{5,1}(y(s=Z)) g_{5,2}\left(y\left(m_{(n T)}, y\left(m_{(n T-T)}\right)\right)\right), \\
(7 . \mathrm{e}) \\
f_{6}(y)=g_{6,1}(y(s=N)) g_{6,2}\left(y\left(m_{(n T)}, y\left(m_{(n T-T)}\right)\right)\right), \\
(7 . f) \\
f_{7}(y)=g_{7,1}(y(s=N G)) g_{7,2}\left(y\left(m_{(n T)}, y\left(m_{(n T-T)}\right)\right)\right) .
\end{array}
$$

Here (7) would be best according to the results of the condition of the trial immediately prior to the current 
trial as this fMRI analysis has shown significant results: Z.G vs. G.G, N.G vs. G.G, NG.G vs. Z.G, NG.G vs. G.G, and N.G vs. Z.G. From (7.a-d) contrast difference found may be accounted by contrast $\left(f_{2}(y), f_{1}(y)\right)$, contrast $\left(f_{3}(y), f_{1}(\mathrm{y})\right)$, contrast $\left(f_{4}(y), f_{1}(y)\right)$, contrast $\left(f_{3}(y), f_{2}(y)\right)$, contrast $\left(f_{2}(y), f_{3}(y)\right)$. An extension of this proposal clearly considers features on signals, where features can be stimulus properties as well.

Also, EEG research may use formulation by (7) on findings considering previous and later interventions on videogames on spectral ERP for fortress hits, rare tones (inside and outside the game), and mine appearances [43]. Limitation here is for a variety of complex and non/complex tasks maybe worked [44].

Main limitation for this proposal is to ignore possible conflict when one tends to think about a bad previous response. In the present experiment, errors were less than $10 \%$ in most of the participants, moreover not different for having more contextual variable are not accounted by $f_{5}(y)$ (equivalent to $\left.f_{2}(y)\right), f_{6}(y)$ (equivalent to $\left.f_{3}(y)\right)$ and $f_{7}(y)$ (equivalent to $f_{4}(y)$ ), of course more experiments should be done to account properly how multitask and prior context affects other conditions. This would open to study motor response with error response in decision-making responses and improve current learning systems in BCI.
This motor response recruiting prefrontal areas would support the idea that the learning modelling of the task has not a linear function influenced by the learning parameter, the greater the maze size for goaltask the more steps to get an optimal pathway [45]. Moreover, the model proposed may help in the future to find compensatory effects in Parkinson's disease by recruitment of more brain area in the prefrontal cortex and extend not only the present work but also work of Martin and colleagues at planning and executing motor employing different hands might be studied simplifying their experiment with an additional condition of motor planning [46]. In this way dopamine pathway can be revisited, having (7) in frequency may help to study beta frequencies in Parkinson at synchronization of the basal ganglia (BG) and thalamus wit cortex [47] as well as a less studied dopamine interpretation for anaemia in children [48]. Impaired motor function would be described as a change con $g_{i, 2}(i=\{1,2, . ., 7)$ and current treatment experiments such as DOPA-ON and ON-Deep Brain Stimulation where the higher duration the longer beta peaks in patients OFF medication (peak width at half height, $106 \mathrm{~ms}$ ) compared to controls (peak width, $46 \mathrm{~ms}$ ) [49].

Limitation for motor response in the present research was about the extension of motor control in the research

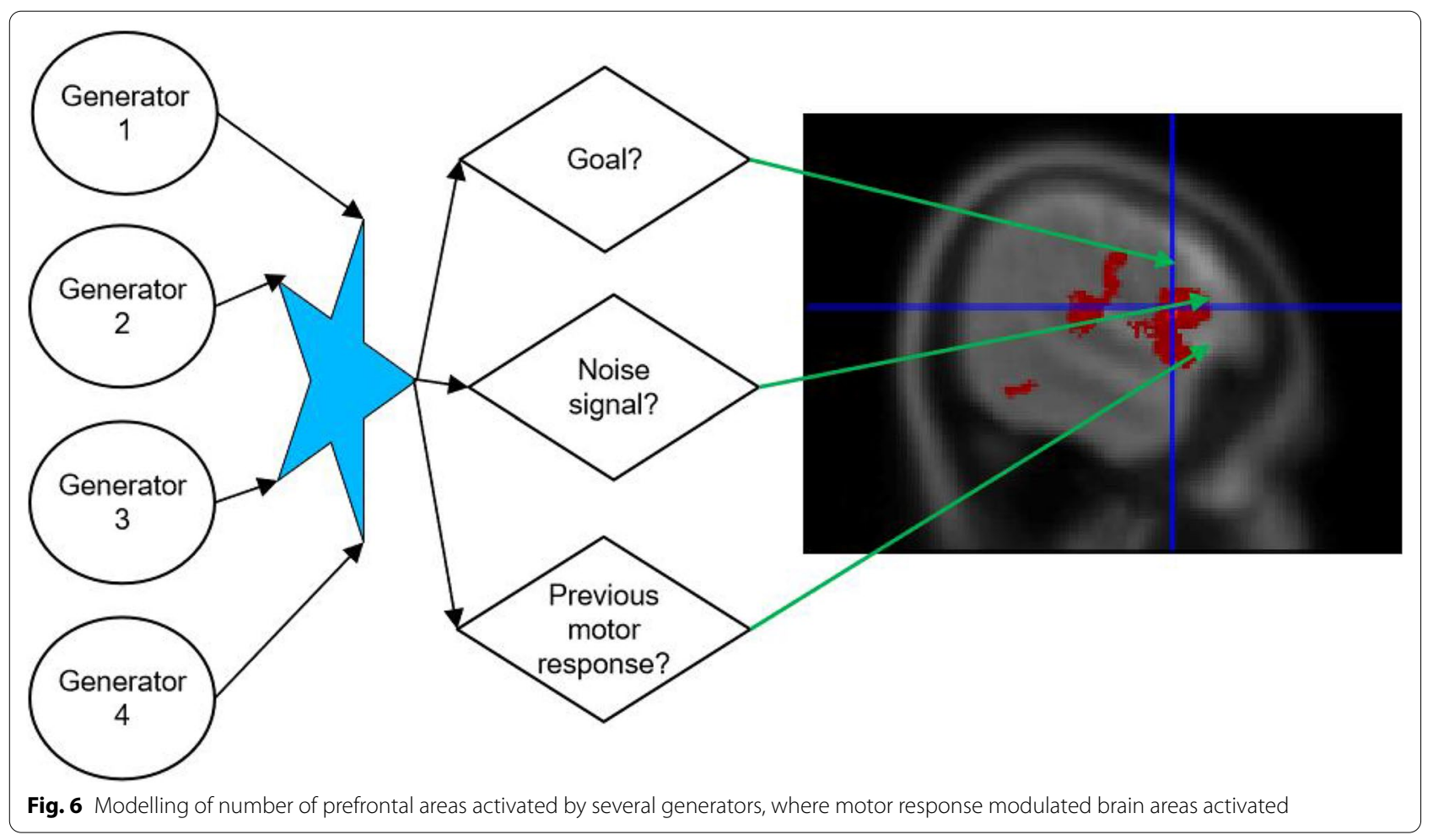


area of "coordination". Marsh and colleagues pointed perception-action systems come to task of ecosystems [50], therefore considering multi-stability for social behaviour and multiple participants present in several real setting multitasks [51]. Participants are believed to not only use dopamine pathway to social rewards, but also to context dependence in complex environment where new selections are done base on dynamic interaction of task [52]. Although the present work has given a better insight of auditory multitask and motor control, it did not reach a real setting multitask, therefore more work should be done to use multitask in perception-action ecosystems in real world.

Another area of further test may be on multitask switching on dyslexia, considering our results mainly on right Pulvinar which is close to LGN, our experimental results suggest an asymmetry for brain processing. Bearing this result on our auditory number parity decision task, language multitask switch may be explored as well, as LGN asymmetry was reported by proton density with MRI recently by Giraldo-Chilca and Schneider [53]. Moreover, in this study, the different modulation of brain areas in the PFC and its concurrent Pulvinar activation may be related to different "coordination through the Pulvinar's involvement in up-regulating activity" [54]. Therefore, current research would be extended by an experimental design using EEG and fMRI to study PFC and Pulvinar interaction with LGN different frequency bands as a Deep Predictive Learning $[37,55,56]$ as well as TMS has been suggested to improve this understanding in dyslexia as well [37]. On the other hand, a possible extension of the present work may be extending cortical-pulvinar interaction described by Kanai and colleagues [57] in terms of some of the equations developed here, namely (7). Possibly extension of the present experiment for modelling may be used to extend findings on two choice tasks.

Finally, bearing in mind discussion of multitask experiment [3] discussed in use of person identification with reliable decoders [2] and re-identification using different visual views [1] in systems with different interfaces. These interfaces may involve not only EEG, but also precise electrode positions inferred or combined with fMRI or fNIRS as occipital images, as the present work suggests. Moreover, prior context in auditory signals has been related to probability is related in auditory judgment with Hidden Markov Models [58] and therefore to attention and decision-making, next step to setup probabilities in the present research is to study parallel judgment as visual 2D, 3D and Augmented reality is been doing recently with Markov chains [59, 60].

\section{Supplementary Information}

The online version contains supplementary material available at https://doi. org/10.1186/s40708-021-00124-6.

Additional file 1. EEG results and typical fMRI contrasts.

\section{Acknowledgements}

The present authors thank lan Cavin for helping recording in Ninewells Hospital, Cyril Pernet from the University of Edinburgh and to Guillaume Rousselet from the University of Glasgow for helping in initial ideas to design the experiment that was reported in the PhD dissertation at the University of Dundee and the dissertation is available at Dundee digital library (discovery.dundee. ac.uk). Also to MAtthew Stainer, Benjamin Vincent and Victoria Gradin for initial feedback on experiment and to Andrés Mugruza for making think better and attention and motor control.

\section{Authors' contributions}

CAMV helped to design, also recorded, analysed, interpreted the data and wrote most of the manuscript. DDP performed most of design, also recorded, interpreted the data and corrected most of the manuscript. ST recorded, helped to initial processing of the data and corrected the manuscript. ST recorded, helped to initial processing of the data and corrected the manuscript. JM helped to initial design recorded, arranged participants to the MRI and suggest initial processing of the data. AM helped to initial design recorded, arranged participants to the MRI and helped to initial processing of the data. All authors read and approved the final manuscript.

\section{Funding}

This study was funded by SINAPSE (Scottish Imaging Network: A Platform for Scientific Excellence).

C.A.M.V. has received a research grant from SINAPSE for "Spatial and tempora imaging of attention reorienting mechanisms". D. D. P has received research grants from SINAPSE. Also, time for setup final interpretation writing up was due to support for research project "Implementación de Laboratorios: Concepción de Laboratorio de Electrofisiología Cognitiva: ElectroEncefaloGrama (EEG) y realidad aumentada" funding won by "Resolución de Comisión Organizadora N 229-2017-UNTELS" and research duties provided for the first author under "Decreto Supremo N. ${ }^{\circ}$ 003-2018-MINEDU" and also for some time to amend interpretation at University of Delaware under the project "EEG and fMRI studies for dyslexia" in Center for Brain and Biomedical Imaging.

\section{Availability of data and materials}

Program, source code and contrast files in SPM are accessible at cmugruza@ yahoo.com (we are heading to share in some workspace on https://github. $\mathrm{com} / \mathrm{cmugruza} / \mathrm{fMRI}$ priorContext). The human head MRI data set was donated under the condition of anonymity during the filling consent inform of each participant.

\section{Declarations}

\section{Ethics approval and consent to participate}

The study was approved by the Ethics committee of the University of Dundee and all participants gave written informed consent to participate.

Consent for publication

Not applicable.

\section{Competing interests}

The authors declare that the research was conducted in the absence of any commercial or financial relationships that could be construed as a potential conflict of interest. 


\section{Author details}

${ }^{1}$ Grupo de Investigación de Computación Y Neurociencia Cognitiva, Facultad de Ingeniería Y Gestión, Universidad Nacional Tecnológica de Lima Sur UNTELS, Lima, Perú. ${ }^{2}$ Neuroscience and Development Group, Arts and Science, University of Dundee, Dundee, UK. ${ }^{3}$ School of Psychology, University of Lincoln, Lincoln, United Kingdom. ${ }^{4}$ NHS Tayside, Ninewells Hospital, Dundee, UK.

Received: 31 August 2020 Accepted: 21 December 2020 Published online: 21 March 2021

\section{References}

1. Wu L, Wang Y, Gao J, Wang M, Zha ZJ, Tao D. Deep co-attention based comparator for relative representation learning in person re-identification. IEEE Trans Neural Netw Learn Syst. 2020.

2. Kwon OY, Lee MH, Guan C, Lee SW (2019) Subject-independent braincomputer interfaces based on deep convolutional neural networks. IEEE Trans Neural Netw Learn Syst 31(10):3839-3852

3. Mugruza Vassallo CA, Potter DD (2019) Context dependence signature, stimulus properties and stimulus probability as predictors of ERP amplitude variability. Front Hum Neurosci 13:39C

4. Koechlin E, Ody C, Kouneiher F (2003) The architecture of cognitive control in the human prefrontal cortex. Science 302(5648):1181-1185

5. Koechlin E, Summerfield C (2007) An information theoretical approach to prefrontal executive function. Trends Cogn Sci 11(6):229-235

6. C. A. Mugruza-Vassallo (2016) Database methodology for therapy evaluation in auditory schizophrenia disorder based on continuity evolution of symptoms. In: Proceedings of the 8th international conference on information communication and management (ICICM). 2016; 10(1): 298-303. https://doi.org/10.1109/INFOCOMAN.2016.7784261

7. Corbetta M, Shulman GL (2002) Control of goal-directed and stimulusdriven attention in the brain. Nat Rev Neurosci 3(3):201-215

8. Kastner LG, Ungerleider S (2000) Mechanisms of visual attention in the human cortex. Annu Rev Neurosci 23(1):315-341

9. Savalia T, Shukla A, Bapi RS (2016) A unified theoretical framework for cognitive sequencing. Front Psychol 7:1821. https://doi.org/10.3389/ fpsyg.2016.01821

10. Rincon-Gonzalez L, Selen LPJ, Halfwerk K, Koppen M, Corneil BD, Medendorp WP (2016) Decisions in motion: vestibular contributions to saccadic target selection. J Neurophysiol 116(3):977

11. Bakker RS, Selen LP, Medendorp WP (2018) Reference frames in the decisions of hand choice. J Neurophysiol 119(5):1809-1817

12. Toprak S, Navarro-Guerrero N, Wermter S (2018) Evaluating integration strategies for visuo-haptic object recognition. Cogn Comput 10(3):408-425

13. Schneider DM, Nelson A, Mooney R (2014) A synaptic and circuit basis for corollary discharge in the auditory cortex. Nature 513(7517):189

14. Livneh $U$, Zador A (2014) Sensory systems: sound processing takes motor control. Nature 513(7517):180

15. Opitz B, Mecklinger A, Friederici A, Cramon D (1999) The functional neuroanatomy of novelty processing: integrating ERP and $\mathrm{fMRI}$ results. Cereb Cortex 9(4):379-391

16. Strobel A, Debener S, Sorger B et al (2008) Novelty and target processing during an auditory novelty oddball: a simultaneous event-related potential and functional magnetic resonance imaging study. Neuroimage 409(2):869-883

17. Kiehl KA, Stevens MC, Laurens KR et al (2005) An adaptive reflexive processing model of neurocognitive function: supporting evidence from a large scale $(n=100) \mathrm{fMRI}$ study of an auditory oddball task. Neuroimage 25(3):899-915

18. Zeng Y, Zhao Y, Bai J, Xu B (2017) Toward robot self-consciousness (II): brain-inspired robot bodily self model for self-recognition. Cogn Comput 10(2):307-320. https://doi.org/10.1007/s12559-017-9505-1

19. Gupta A, Ong YS (2019) Back to the roots: multi-x evolutionary computation. Cogn Comput 11(1):1-7

20. Corbetta M, Patel G, Shulman GL (2008) 'The reorienting system of the human brain: from environment to theory of'. Mind. Neuron 58(3):306-324
21. Friston K (2004) Introduction to statistical parametric mapping. In: Frackowiak RSJ (ed) Human brain function. Academic Press, London

22. Slotnick SD, Moo LR, Segal JB, Hart J (2003) Distinct prefrontal cortex activity associated with item memory and source memory for visual shapes. Cogn Brain Res 17(1):75-82

23. Sherman SM, Guillery RW (2006) Exploring the thalamus and its role in cortical function. MIT Press, Cambridge, MA

24. Hönegger C, Atteneder C, Griesmayr B, Holz E, Weber E, Sauseng P (2011) Neural correlates of visuo-spatial working memory encoding —an EEG study. Neurosci Lett 500(2):118-122. https://doi.org/10.1016/j.chb.2018. 02.035

25. Van Veen V, Carter CS (2002) The anterior cingulate as a conflict monitor: fMRI and ERP studies. Physiol Behav 77(4):477-482

26. Fan J, McCandliss BD, Fossella J, Flombaum JI, Posner MI (2005) The activation of attentional networks. Neuroimage 26(2):471-479

27. Astafiev SV, Shulman GL, Stanley CM et al (2003) Functional organization of human intraparietal and frontal cortex for attending, looking, and pointing. J Neurosci 23(11):4689-4699

28. Connolly JD, Goodale MA, Menon RS, Munoz DP (2002) Human fMRI evidence for the neural correlates of preparatory set. Nat Neurosci 5(12):1345-1352

29. Inouchi M, Matsumoto R, Taki J et al (2013) Role of posterior parietal cortex in reaching movements in humans: clinical implication for 'optic ataxia.'Clin Neurophysiol 124(11):2230-2241

30. Menéndez-Colino LM, Falcon C, Traserra J et al (2007) Activation patterns of the primary auditory cortex in normal-hearing subjects: a functional magnetic resonance imaging study. Acta oto-laryngol 127(12):1283-1291

31. Binder J, Frost J, Hammeke T et al (2000) Human temporal lobe activation by speech and nonspeech sounds. Cereb Cortex 10(5):512-528

32. Schacter DL, Buckner RL (1998) Priming and the brain. Neuron 20(2):185-195

33. Henson R, Shallice T, Dolan R (2000) Neuroimaging evidence for dissociable forms of repetition priming. Science 287:1269-1272

34. Rinne T, Kirjavainen S, Salonen O et al (2007) Distributed cortical networks for focused auditory attention and distraction. Neurosci Lett 416(3):247-251

35. Gouws AD, Alvarez I, Watson DM, Uesaki M, Rogers J, Morland AB (2014) On the role of suppression in spatial attention: evidence from negative BOLD in human subcortical and cortical structures. J Neurosci 34(31):10347-10360

36. Zhou H, Schafer RJ, Desimone R (2016) Pulvinar-cortex interactions in vision and attention. Neuron 89(1):209-220. https://doi.org/10.1016/j. neuron.2015.11.034

37. Mugruza-Vassallo CA, Schneider KA (2020) Rhyme and rhythm modulation in dyslexia. BrinBox Conference; 2020. http://brainbox-initiative. com/wp-content/uploads/2020/09/RhYme-and-rhytm-modulation-indyslexia.pdf

38. D. D. Potter, A. Schloerscheidt, D. Back et al. Development of Translational Biomarkers of Attention Function. Glasgow, 2008.

39. Matsuda T, Matsuura M, Ohkubo T et al (2002) Influence of arousal level for functional magnetic resonance imaging (fMRI) study: simultaneous recording of fMRI and electroencephalogram. Psychiatry Clin Neurosci 56(3):289-290

40. Nichols T, Hayasaka S (2003) Controlling the familywise error rate in functional neuroimaging: a comparative review. Stat Meth in Med Research 12(5):419-446

41. Fink LK, Hurley BK, Geng JJ, Janata P (2018) A linear oscillator model predicts dynamic temporal attention and pupillary entrainment to rhythmic patterns. J Eye Movement Res 11(2):1-24. https://doi.org/10. 16910/jemr.11.2.12

42. Forth J, Agres K, Purver M, Wiggins GA (2016) Entraining IDyOT: timing in the information dynamics of thinking. Front Psychol 7:1575

43. Maclin EL, Mathewson KE, Low KA, Boot WR, Kramer AF, Fabiani M, Gratton G (2011) Learning to multitask: effects of video game practice on electrophysiological indices of attention and resource allocation. Psychophysiology 48(9):1173-1183

44. Liu S, Nam CS (2018) Quantitative modeling of user performance in multitasking environments. Comput Hum Behav 84:130-140

45. Mugruza-Vassallo C, Rivero T (2018) The role of the size maze and learning parameters in the prefrontal cortex modeling based in minicolumns. In: Proceedings of the 8 th international conference on 
information communication and management. 10(1): 67-72. ACM. https://doi.org/10.1145/3268891.3268901

46. Martin JA, Zimmermann N, Scheef L, Jankowski J, Paus S, Schild HH et al (2019) Disentangling motor planning and motor execution in unmedicated de novo Parkinson's disease patients: an fMRI study. Neurolmage Clin 22:101784

47. Sharott A, Gulberti A, Zittel S, Jones AAT, Fickel U, Münchau A et a (2014) Activity parameters of subthalamic nucleus neurons selectively predict motor symptom severity in Parkinson's disease. J Neurosc 34(18):6273-6285

48. Algarín Crespo CR, Nelson CA, Peirano Campos P, Westerlund E, Reyes S, Lozoff B (2013) Iron-deficiency anemia in infancy and poorer cognitive inhibitory control at age 10 years. Dev Med Child Neurol 55(5):453-458

49. Gulberti A, Moll CKE, Hamel W, Buhmann C, Koeppen JA, Boelmans K, Zittel S, Gerloff C, Westphal M, Schneider TR, Engel AK (2015) Predictive timing functions of cortical beta oscillations are impaired in Parkinson's disease and influenced by L-DOPA and deep brain stimulation of the subthalamic nucleus. Neurolmage Clin 9:436-449

50. Marsh K, Johnston L, Richardson MJ, Schmidt RC (2009) Toward a radically embodied, embedded social psychology. Eur I Soc Psychol. https://doi.org/10.1002/ejsp.666

51. Marsh K, Richardson MJ, Baron RM, Schmidt RC (2010) Contrasting approaches to perceiving and acting with others. Ecol Psychol 18(1):1-38. https://doi.org/10.1207/s15326969eco1801_1

52. Voyer BG, Franks B (2014) Toward a better understanding of self-construal theory: an agency view of the processes of self-construal. Rev Gen Psychol 18(2):101-114

53. Giraldo-Chica M, Schneider KA (2018) Hemispheric asymmetries in the orientation and location of the lateral geniculate nucleus in dyslexia. Dyslexia 24(2):197-203

54. Green SA, Hernandez L, Bookheimer SY, Dapretto M (2017) Reduced modulation of thalamocortical connectivity during exposure to sensory stimuli in ASD. Autism Res 10(5):801-809

55. Barczak A, O'Connell MN, McGinnis T, Ross D, Mowery T, Falchier A, Lakatos $P$ (2018) Top-down, contextual entrainment of neuronal oscillations in the auditory thalamocortical circuit. Proc Natl Acad Sci 115(32):E7605E7614. https://doi.org/10.1073/pnas.1714684115
56. O'Reilly RC, Russin JL, Zolfaghar M, Rohrlich J (2020) Deep predictive learning in neocortex and pulvinar. arXiv preprint. arXiv 2006.14800

57. Kanai R, Komura Y, Shipp S, Friston K (2015) Cerebral hierarchies: predictive processing, precision and the pulvinar. Philos Trans R Soc B Biol Sci 370(1668):20140169

58. Chambers C, Akram S, Adam V, Pelofi C, Sahani M, Shamma S, Pressnitzer D (2017) Prior context in audition informs binding and shapes simple features. Nat Commun 8(1):1-11

59. Torres-Tejeda S, Portilla-Fernández JA, Mugruza-Vassallo CA, Córdoba-Berrios LL (2020) RVariations of reaction times explained by stimuli changes in size and perspective in 2D and 3D for selective attention. Revista mexicana de ingeniería biomédica 41(1):91-104

60. Mugruza-Vassallo CA, Granados-Domínguez JL, Flores-Benites V, Córdoba-Berrios LL (2020) Markov Response to visual stimuli in a Go-Go experiment in 2D, 3D and augmented reality

61. Simmonds DJ, Pekar JJ, Mostofsky SH (2008) Meta-analysis of Go/No-go tasks demonstrating that $\mathrm{fMRI}$ activation associated with response inhibition is task-dependent. Neuropsychologia 46(1):224-232

62. Sato M, Hikosaka O (2002) Role of primate substantia nigra pars reticulata in reward-oriented saccadic eye movement. J Neurosci 22(6):2363-2373

63. Hikosaka O, Yamamoto S, Yasuda M, Kim HF (2013) Why skill matters. Trends Cogn Sci 17(9):434-441

64. Krall S, Rottschy C, Oberwelland E et al (2015) The role of the right temporoparietal junction in attention and social interaction as revealed by ALE meta-analysis. Brain Struct Funct 220(2):587-604

65. Weissman D, Giesbrecht B, Song A, Mangun G, Woldorff M (2003) Conflict monitoring in the human anterior cingulate cortex during selective attention to global and local object features. Neuroimage 19(4):1361-1368

66. Polich J (2007) Updating P300: an integrative theory of P3a and P3b. Clin neurophysiol 118(10):2128-2148

67. Stevens A, Skudlarski P, Gatenby J, Gore JC (2000) Event-related fMRI of auditory and visual oddball tasks. Magn Reson Imaging 18(5):495-502

68. Roelfsema PR, Holtmaat A (2018) Control of synaptic plasticity in deep cortical networks. Nat Rev Neurosci 19(3):166

\section{Publisher's Note}

Springer Nature remains neutral with regard to jurisdictional claims in published maps and institutional affiliations. 